\title{
A Detailed Study of the Gluino Decay into the Third Generation Squarks at the CERN LHC
}

\author{
Junji Hisano, ${ }^{1}$ Kiyotomo Kawagoe, ${ }^{2}$ and Mihoko M. Nojiri ${ }^{3}$ \\ ${ }^{1}$ ICRR, University of Tokyo, Kashiwa 277-8582, Japan \\ ${ }^{2}$ Department of Physics, Kobe University, Kobe 657-8501, Japan \\ ${ }^{3}$ YITP, Kyoto University, Kyoto 606-8502, Japan
}

(Dated: October 29, 2018)

\begin{abstract}
In supersymmetric models a gluino can decay into $t b \tilde{\chi}_{1}^{ \pm}$through a stop or a sbottom. The decay chain produces an edge structure in the $m_{t b}$ distribution. Monte Carlo simulation studies show that the end point and the edge height would be measured at the CERN LHC by using a sideband subtraction technique. The stop and sbottom masses as well as their decay branching ratios are constrained by the measurement. We study interpretations of the measurement in the minimal supergravity model. We also study the gluino decay into $t b \tilde{\chi}_{2}^{ \pm}$as well as the influence of the stop left-right mixing on the $m_{b b}$ distribution of the tagged $t b$ events.
\end{abstract}

PACS numbers: 12.60.Jv, 14.80.Ly

\section{INTRODUCTION}

The minimal supersymmetric standard model (MSSM) is one of the promising extensions of the standard model [1]. The model requires superpartners of the standard model particles (sparticles), and the large hadron collider (LHC) at CERN might confirm the existence of the new particles [2]. The LHC is a $p p$ collider at $\sqrt{s}=14 \mathrm{TeV}$, whose operation is currently expected to start in 2007 . The integrated luminosity will be $10 \mathrm{fb}^{-1} /$ year at the beginning (low luminosity runs), and then upgraded to $100 \mathrm{fb}^{-1}$ /year (high luminosity runs).

Supersymmetry (SUSY) must be broken and the sparticle mass spectrum depends on the SUSY breaking mechanism. Measurement of the sparticle masses provides a way to probe the origin of the SUSY breaking in nature. The sparticle mass measurement at the LHC has been therefore extensively studied.

Among the sparticles the third generation squarks, stops $\left(\tilde{t}_{i}\right)$ and sbottoms $\left(\tilde{b}_{i}\right)(i=1,2)$, get special imprints from physics at the very high energy scale. One of the examples is found in a model with universal scaler masses. Although the scalar masses are universal at a high energy scale, the third generation squarks are much lighter than the first and second generation squarks due to the Yukawa running effect. On the other hand, some SUSY breaking models, such as the flavor U(2) model [3] or the decoupling solution [4], and the superconformal model [5] for the SUSY flavor problem, have nonuniversal boundary conditions for the third generation mass parameters at the GUT scale. In addition, the $\tilde{t}_{L}-\tilde{t}_{R}$-Higgs trilinear coupling $A_{t}$ is comparable to the gluino mass at the weak scale when the origin of the SUSY breaking comes from the GUT scale or the Planck scale physics. This appears in the stop mass matrix with a large coefficient $m_{t} ; m_{L R}^{2}=m_{t}\left(A_{t}-\mu \cot \beta\right)$. The stop left-right mixing is therefore expected to be sizable, leading to an even lighter stop mass compared to those of other squarks [6]. It should be stressed that the stop masses and the mixing are very important parameters to predict the light Higgs mass [7], or the rare $B$ decay ratios [8].

We may be able to access the nature of the stop and sbottom at the LHC provided that they are lighter than the gluino $(\tilde{g})$. They may copiously arise from the gluino decay if the decay is kinematically allowed. The relevant decay modes for $\tilde{b}_{i}(i=1,2), \tilde{t}_{1}$, to charginos $\tilde{\chi}_{j}^{ \pm}(j=$ 1,2) or neutralinos $\tilde{\chi}_{j}^{0}(j=1,2,3,4)$ are listed below (indices to distinguish a particle and its anti-particle is suppressed unless otherwise stated)[21],

$$
\begin{aligned}
(\mathrm{I})_{j} & \tilde{g} \rightarrow b \tilde{b}_{1} \rightarrow b b \tilde{\chi}_{j}^{0}\left(\rightarrow b b l^{+} l^{-} \tilde{\chi}_{1}^{0}\right), \\
(\mathrm{II})_{j} & \tilde{g} \rightarrow t \tilde{t}_{1} \rightarrow t t \tilde{\chi}_{j}^{0} \\
(\mathrm{III})_{j} & \tilde{g} \rightarrow t \tilde{t}_{1} \rightarrow t b \tilde{\chi}_{j}^{ \pm} \\
(\mathrm{III})_{i j} & \tilde{g} \rightarrow b \tilde{b}_{i} \rightarrow b W \tilde{t}_{1} \rightarrow b b W \tilde{\chi}_{j}^{ \pm} \\
(\mathrm{IV})_{i j} & \tilde{g} \rightarrow b \tilde{b}_{i} \rightarrow t b \tilde{\chi}_{j}^{ \pm} .
\end{aligned}
$$

In previous literatures $[2,9]$, the lighter sbottom $\tilde{b}_{1}$ is often studied through the mode $(\mathrm{I})_{2}$, namely the $b b \tilde{\chi}_{2}^{0} \rightarrow$ $b b l^{+} l^{-} \tilde{\chi}_{1}^{0}$ channel. This mode is important when the second lightest neutralino $\tilde{\chi}_{2}^{0}$ has substantial branching ratios into leptons. The difference of sparticle masses such as $\left(m_{\tilde{g}}-m_{\tilde{b}_{1}}\right)$ is determined by measuring kinematical end points of invariant mass distributions for signal events.

In a previous paper [10] we proposed to measure the edge position of the $m_{t b}$ distribution for the modes (III) $)_{1}$ and (IV $)_{11}$, where $m_{t b}$ is the invariant mass of a top-bottom $(t b)$ system. The decay modes are expected to be dominant in the minimal supergravity model (MSUGRA), since the branching ratios $\operatorname{Br}\left(\tilde{b}_{1}\left(\tilde{t}_{1}\right) \rightarrow\right.$ $\left.t(b) \tilde{\chi}_{1}^{ \pm}\right)$could be as large as $60 \%$. We focused on the reconstruction of hadronic decays of the top quark, because the $m_{t b}$ distribution of the decay makes a clear "edge" in this case. The parton level $m_{t b}$ distributions for the modes (III) ${ }_{j}$ and (IV) $)_{i j}$ are expressed as functions of $m_{\tilde{g}}$, $m_{\tilde{t}_{1}}, m_{\tilde{b}_{i}}$, and the chargino mass $m_{\tilde{\chi}_{j}^{ \pm}}: d \Gamma / d m_{t b} \propto m_{t b}$, and the edge position (end point) of the $m_{t b}$ distribution 
$M_{t b}$ for the modes (III) $)_{j}$ and (IV) $)_{i j}$ are written as follows;

$$
\begin{aligned}
& M_{t b}^{2}(\mathrm{III})_{j}=m_{t}^{2}+\frac{m_{\tilde{t}_{1}}^{2}-m_{\tilde{\chi}_{j}^{ \pm}}^{2}}{2 m_{\tilde{t}_{1}}^{2}}\left\{\left(m_{\tilde{g}}^{2}-m_{\tilde{t}_{1}}^{2}-m_{t}^{2}\right)\right. \\
& \left.+\sqrt{\left(m_{\tilde{g}}^{2}-\left(m_{\tilde{t}_{1}}-m_{t}\right)^{2}\right)\left(m_{\tilde{g}}^{2}-\left(m_{\tilde{t}_{1}}+m_{t}\right)^{2}\right)}\right\} \\
& M_{t b}^{2}(\mathrm{IV})_{i j}=m_{t}^{2}+\frac{m_{\tilde{g}}^{2}-m_{\tilde{b}_{i}}^{2}}{2 m_{\tilde{b}_{i}}^{2}}\left\{\left(m_{\tilde{b}_{i}}^{2}-m_{\tilde{\chi}_{j}^{ \pm}}^{2}+m_{t}^{2}\right)\right. \\
& \left.+\sqrt{\left(m_{\tilde{b}_{i}}^{2}-\left(m_{\tilde{\chi}_{j}^{ \pm}}-m_{t}\right)^{2}\right)\left(m_{\tilde{b}_{i}}^{2}-\left(m_{\tilde{\chi}_{j}^{ \pm}}+m_{t}\right)^{2}\right)}\right\}
\end{aligned}
$$

The measurement of the end points is sensitive to both $m_{\tilde{t}_{1}}$ and $m_{\tilde{b}_{1}}$, provided that $m_{\tilde{g}}$ and $m_{\tilde{\chi}^{ \pm}}$are determined from other measurements. The edge height is then closely related to $\sigma(p p \rightarrow \tilde{g} X) \times \operatorname{Br}(\mathrm{III} / \mathrm{IV})$. In some model parameters $M_{t b}(\mathrm{III})_{1}$ is very close to $M_{t b}(\mathrm{IV})_{11}$. When they are experimentally indistinguishable, it is convenient to define a weighted mean of the end points;

$$
\begin{aligned}
M_{t b}^{\mathrm{w}} & =\frac{\operatorname{Br}(\mathrm{III}) M_{t b}(\mathrm{III})_{1}+\operatorname{Br}(\mathrm{IV})_{11} M_{t b}(\mathrm{IV})_{11}}{\operatorname{Br}(\mathrm{III})+\operatorname{Br}(\mathrm{IV})_{11}}, \\
\operatorname{Br}(\mathrm{III}) & \equiv \operatorname{Br}(\mathrm{III})_{1}+\operatorname{Br}(\mathrm{III})_{11}+\operatorname{Br}(\mathrm{III})_{21} .
\end{aligned}
$$

As the final states $b b W$ from the decay chain $\tilde{g} \rightarrow b \tilde{b}_{i} \rightarrow$ $b W \tilde{t}_{1} \rightarrow b b W \tilde{\chi}_{1}^{ \pm}$(mode (III) $)_{i 1}$ ) could have an irreducible contribution to the $t b$ final state, they are included in the definition of $M_{t b}^{\mathrm{w}}$.

In the previous paper we showed that the weighted mean $M_{t b}^{\mathrm{w}}$ was successfully measured from a fit to the $m_{t b}$ distribution. We also discussed the interpretation of the measurement. In this paper we significantly extend our study to the branching ratio measurements, identification of the stop and sbottom decays into heavier charginos, and the interpretation in the MSUGRA model.

This paper is organized as follows. In Section II, we explain our reconstruction and fitting procedure of the edge structure in detail. We use a sideband method to estimate the background distribution due to misreconstructed events, which plays a key role in the edge reconstruction. Monte Carlo simulations show that the distribution of the signal modes (III) and (IV) after subtracting the sideband background is very close to the parton level distribution. In Section III, we explore the MSUGRA parameter space to check if our fitting procedure reproduces the end point $M_{t b}^{\mathrm{w}}$ and the number of events going through the decay modes (III) and (IV). We then turn into more delicate issues such as extraction of branching ratios. In Section IV, we discuss the stop and sbottom decays into the heavier chargino $\tilde{\chi}_{2}^{ \pm}$by looking into the $m_{t b}$ distribution of events with additional leptons. In Section V, we study the MSUGRA parameter region where the decay modes (III) and/or (IV) are open, and discuss how to extract the fundamental parameters using the $m_{t b}$ distributions. The LHC's potential to extract the top polarization arising from the decay $\tilde{g} \rightarrow t \tilde{t}_{1}$ is discussed in Section VI. Section VII is devoted to conclusions.

\section{SIMULATION AND RECONSTRUCTION}

In the MSUGRA model the sparticle spectrum is parameterized by the universal scalar mass $m_{0}$, the universal gaugino mass $M_{0}$, the trilinear coupling of the scalar fields $A_{0}$ at the GUT scale $\left(M_{G U T}\right)$, the ratio of the vacuum expectation values $\tan \beta$, and sign of the higgsino mass parameter $\mu$. In order to demonstrate the end point reconstruction, we take an MSUGRA point with $m_{0}=100 \mathrm{GeV}, M_{0}=300 \mathrm{GeV}, A_{0}=-300 \mathrm{GeV}$, $\tan \beta=10$ and $\mu>0$. This corresponds to the sample point A1 in Table I. The masses and mixings of sparticles are calculated by ISASUSY/ISASUGRA 7.51 [11]. Two different event generators, PYTHIA 6.1 [12] and HERWIG 6.4 [13], are utilized to generate Monte Carlo SUSY events using the masses and mixings. In this section we show results with PYTHIA. The events are then passed through a fast detector simulation program for the ATLAS experiment, ATLFAST [14]. This program performs jet reconstruction in the calorimeters and momentum/energy smearing for leptons and photons, giving a list of reconstructed jets as well as identified leptons and photons. We generate a total of $3 \times 10^{6}$ SUSY events, which correspond to an integrated luminosity of $120 \mathrm{fb}^{-1}$. In addition to the SUSY events, $t \bar{t}$ events are generated using PYTHIA as the standard model background. The number of generated events amounts to $1.94 \times 10^{8}$, corresponding to an integrated luminosity of $286 \mathrm{fb}^{-1}$. In the plots in this paper, the $t \bar{t}$ background is not included unless otherwise noted.

In the detector simulation, a lepton is identified if $p_{T}>$ $5 \mathrm{GeV}$ and $|\eta|<2.5$ for an electron, and $p_{T}>6 \mathrm{GeV}$ and $|\eta|<2.5$ for a muon, respectively. A lepton is regarded as isolated if it is separated by $R>0.4$ from other calorimeter clusters and the transverse calorimeter energy in a cone size $R=0.2$ around the lepton is less than $10 \mathrm{GeV}$. The cone size is defined as $R=\sqrt{(\Delta \phi)^{2}+(\Delta \eta)^{2}}$, where $\phi$ and $\eta$ are the azimuthal angle and the pseudo-rapidity, respectively.

By default, jets are reconstructed by a cone-based algorithm with a cone size $R=0.4$ in the detector simulation [22]. After applying the algorithm, jets having a transverse energy $\left(E_{T}\right)$ more than $10 \mathrm{GeV}$ are kept as reconstructed jets. The $b$ and $\tau$ tagging efficiencies are set to be $60 \%$ and $50 \%$, respectively. The energy of the reconstructed jet is recalibrated according to its jet flavor using a parameterization optimized to give a proper scale of the dijet mass.

First, we apply the following selection cuts to the simulated events for the $t b$ signal:

1. The missing transverse energy $E_{T}^{\text {miss }}$ is greater than $200 \mathrm{GeV}$, where $E_{T}^{\text {miss }}$ is calculated from the reconstructed jets, leptons, photons, and unreconstructed calorimeter energies. This calculation is performed before the recalibration of jet energies.

2. The effective mass $m_{\text {eff }}$ is greater than $1000 \mathrm{GeV}$, where $m_{\mathrm{eff}}$ is the sum of the missing transverse 


\begin{tabular}{|c|c|c|c|c|c|c|}
\hline & $m_{\tilde{g}}$ & $m_{\tilde{t}_{1}}$ & $m_{\tilde{b}_{1}}$ & $m_{\tilde{b}_{2}}$ & $m_{\tilde{\chi}^{ \pm}}$ & $\sigma_{S U S Y}$ \\
\hline $\mathrm{A} 1$ & 707 & 427 & 570 & 613 & 220 & 26 \\
$\mathrm{~A} 2$ & 706 & 496 & 587 & 614 & 211 & 25 \\
$\mathrm{~T} 1$ & 707 & 327 & 570 & 613 & 220 & 30 \\
$\mathrm{~T} 2$ & 707 & 477 & 570 & 612 & 211 & 25 \\
\hline $\mathrm{B}$ & 609 & 402 & 504 & 534 & 179 & 56 \\
$\mathrm{C}$ & 931 & 636 & 771 & 805 & 304 & 5 \\
$\mathrm{G}$ & 886 & 604 & 714 & 763 & 285 & 7 \\
$\mathrm{I}$ & 831 & 571 & 648 & 725 & 265 & 10 \\
\hline $\mathrm{E} 1$ & 515 & 273 & 521 & 634 & 153 & 77 \\
$\mathrm{E} 2$ & 747 & 524 & 770 & 898 & 232 & 8 \\
\hline
\end{tabular}

TABLE I: Sparticle masses in GeV and the total SUSY cross section $\left(\sigma_{S U S Y}\right)$ in $\mathrm{pb}$ for the parameter points studied in this paper. The cross sections are calculated by PYTHIA.

energy and transverse momentum of reconstructed jets, namely $m_{\mathrm{eff}}=E_{T}^{\mathrm{miss}}+\sum_{\text {all }} p_{T}^{\text {jet }}$.

3. There are two and only two $b$-jets with $p_{T}>$ $30 \mathrm{GeV}$ in an event.

4. Excluding the two $b$-jets and those identified as taujets, the number of remaining reconstructed jets with $p_{T}>30 \mathrm{GeV}$ and $|\eta|<3.0$ should be between four and six, inclusive.

Distributions of the cut variables are shown in Fig. 1. The first two cuts are to enhance the SUSY events against the standard model background events. The other two cuts are to reduce combinatorial background (wrong combinations of jets) in the reconstruction of the top quark.

To reconstruct the hadronic decay of the top quark, we take the following steps (i)-(iv):

(i) We first take jet pairs consistent with a hadronic $W$ boson decay with a cut on the jet pair invariant mass $m_{j j}:\left|m_{j j}-m_{W}\right|<15 \mathrm{GeV}$. The $m_{j j}$ distribution is shown in Fig. 2(a), where the selected mass region is marked as $\mathbf{W}$ (the $W$ mass region). Although fake $W$ pairs dominate the distribution, a small bump due to real $W$ bosons can be seen in the mass region.

(ii) The invariant mass of the jet pair and one of the $b$-jets, $m_{b j j}$, is then calculated. All possible combinations of jet pairs and $b$-jets are tried in an event to select the combination which minimizes the difference $\left|m_{b j j}-m_{t}\right|$. The distribution of the selected $m_{b j j}$ is shown in Fig. 3(a).

(iii) The energy and momentum of the jet pair are scaled so that $m_{j j}=m_{W}$, and the invariant mass $m_{b j j}$ is recalculated. The distribution is shown in Fig. 3(b). The jet combination is regarded as a top quark candidate if $\left|m_{b j j}-m_{t}\right|<30 \mathrm{GeV}$.
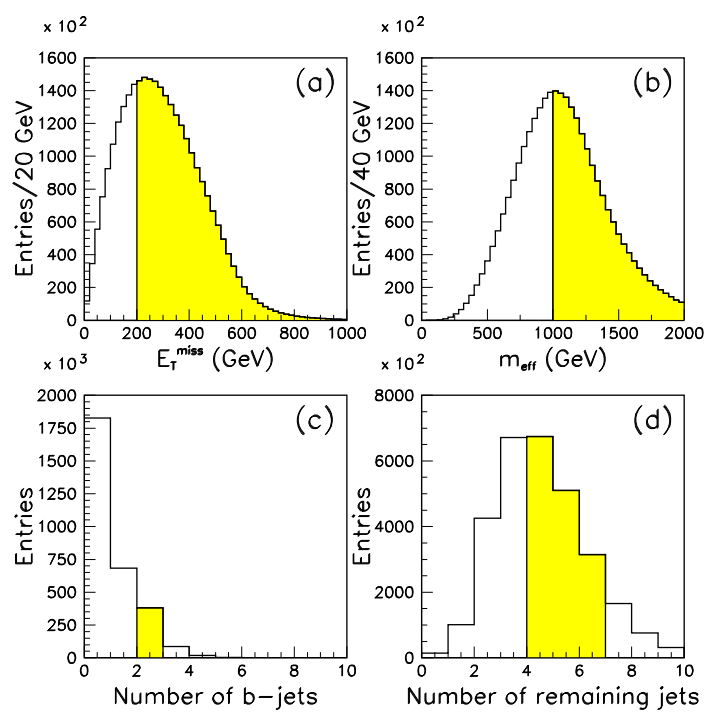

FIG. 1: Distributions of (a) missing transverse energy, (b) effective mass, (c) number of $b$-jets, and (d) number of remaining jets, for $3 \times 10^{6}$ SUSY events at the point A1. Accepted regions are hatched.

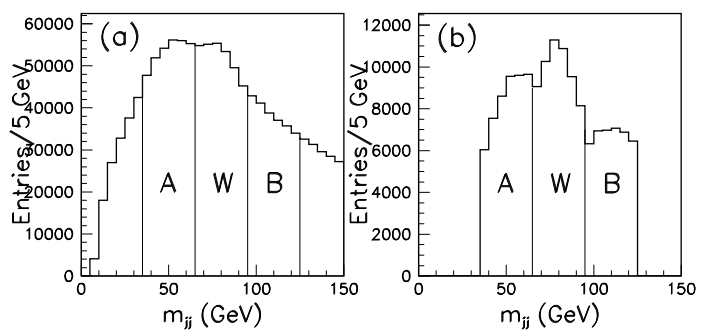

FIG. 2: Distributions of the invariant mass of two jets (a) for all possible combinations, and (b) for the combination used to reconstruct a top candidate. $\mathbf{W}$ and $\mathbf{A}, \mathbf{B}$ indicate the $W$ mass region and the $W$ sidebands, respectively.

As jets are supplied from gluino or squark decays and there are several jets in a selected event, events with a fake $W$ boson (a jet pair that accidentally has $\left.m_{j j} \sim m_{W}\right)$ still dominate the $m_{b j j}$ distribution. The contribution of the fake $W$ boson in the $W$ mass region is estimated from the events that contain jet pairs with the invariant mass in the regions $\mathbf{A}:\left|m_{j j}-\left(m_{W}-30 \mathrm{GeV}\right)\right|<$ $15 \mathrm{GeV}$ and $\mathbf{B}:\left|m_{j j}-\left(m_{W}+30 \mathrm{GeV}\right)\right|<15 \mathrm{GeV}$. We call them "the $W$ sidebands". The energy and momentum of the jet pairs are then scaled linearly to be in the $W$ mass region $\left|m_{j j}-m_{W}\right|<15 \mathrm{GeV}$. The $m_{j j}$ distributions before the linear scaling are shown for the $W$ region and the $W$ sidebands in Fig. 2(b). The distribution of the fake top quark candidates is estimated by following the same steps (ii)-(iv) for the scaled jet pair (see the hatched histogram in Fig. 3(c)). The "true" dis- 
tribution is obtained by subtracting the background distribution estimated by the $W$ sidebands from the original distribution (see Fig. 3(d)). The estimation is based on an assumption that the background comes from the jets without significant correlation with the $b$-jets.
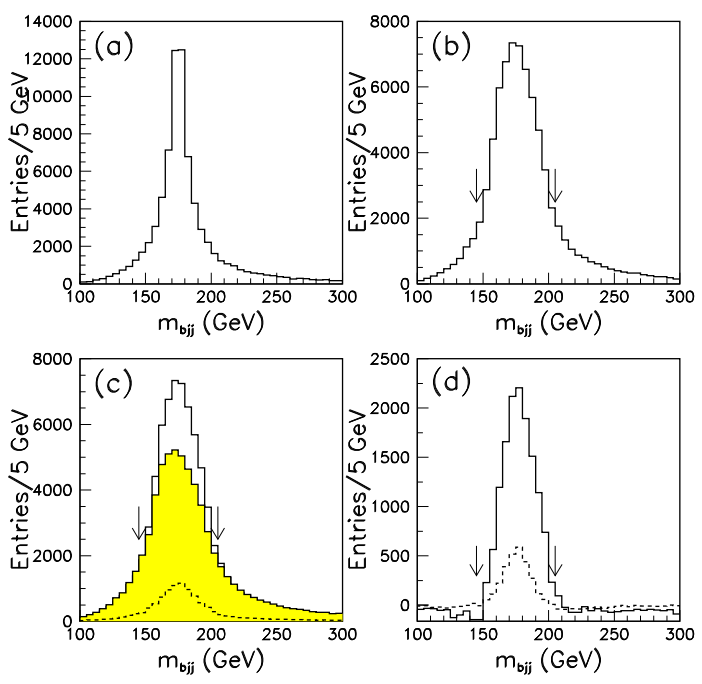

FIG. 3: Distributions of $m_{b j j}$ (a) before and (b) after the jet energy scaling, (c) background estimated by the $W$ sidebands (the hatched histogram) superimposed to the distribution (b), and (d) after subtracting the estimated background. The mass cut for the top candidate is indicated by arrows. Dashed histograms in (c) and (d) show the distributions of $t \bar{t}$ events, where the integrated luminosity of the $t \bar{t}$ events is normalized to that of the SUSY events.

The $t \bar{t}$ production is the dominant standard model background. The $m_{b j j}$ distributions of the $t \bar{t}$ events surviving at this stage are shown in Fig. 3(c) and (d) before and after the sideband background subtraction, respectively. The integrated luminosity of the $t \bar{t}$ events is normalized to that of the SUSY events. To reduce the $t \bar{t}$ events, one of the following two lepton cuts may be used, depending on the signal/background situation.

- Loose lepton cut: If there are high- $p_{T}$ isolated leptons, the invariant mass of any high- $p_{T}$ lepton and the remaining $b$-jet $\left(m_{b l}\right)$ should be greater than $150 \mathrm{GeV}$. The distribution of the minimum $m_{b l}$ in events with a top candidate is shown in Fig. 4.

- Hard lepton cut: An event should have no isolated leptons.

The cuts reduce the fraction of the events having a $t \bar{t}$ pair, where one of the top quarks decays leptonically. The $m_{b j j}$ distributions with the lepton cuts are shown in Fig. 5. The numbers of selected events are listed in Table II. Hereafter we use the loose lepton cut.

For the remaining events, the top candidate is then combined with the other $b$-jet, which is not used to reconstruct the top candidate, to calculate the invariant

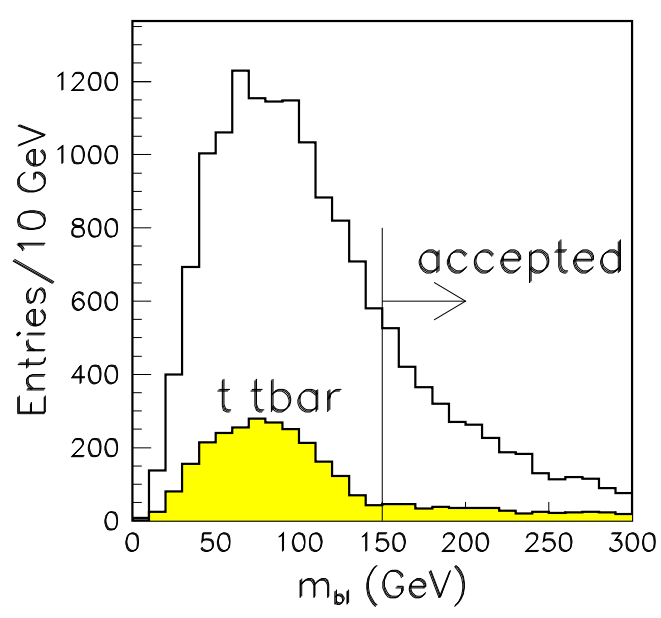

FIG. 4: Distribution of minimum $m_{b l}$ for events having a top candidate. Open and hatched histograms are for SUSY events and $t \bar{t}$ events, respectively. The loose lepton cut is also shown.
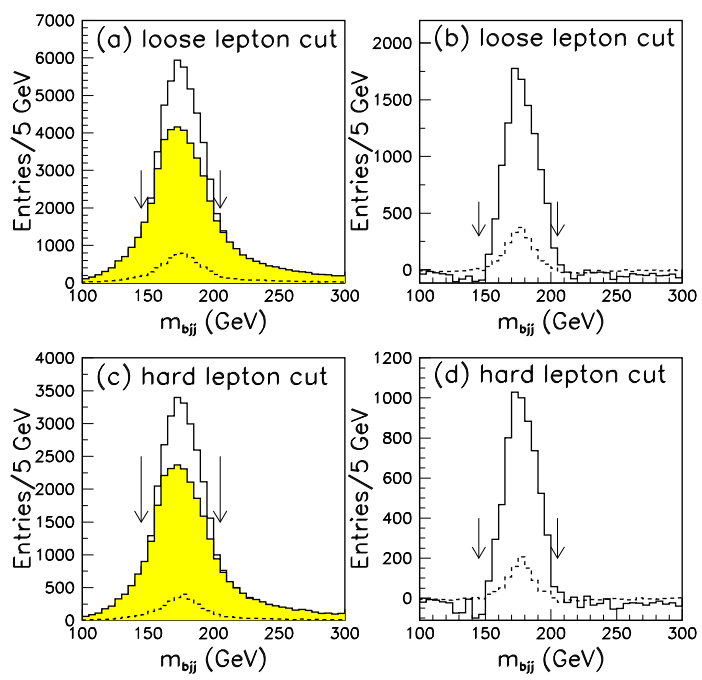

FIG. 5: (a) and (b): Distributions of $m_{b j j}$ with the loose lepton cut before and after sideband subtraction, respectively. (c) and (d) : Distributions of $m_{b j j}$ with the hard lepton cut before and after the sideband subtraction. The meaning of the histograms is as same as in Fig. 3(c) and (d).

mass of the $t b$ system $m_{t b}$. The distribution is shown in Fig. 6(a). However, the expected $t b$ end point is not clearly visible in the $m_{t b}$ distribution due to the fake $W$ events. Here we can again utilize the $W$ sidebands for the background estimation. The estimated background distribution is shown in Fig. 6(b), which is obtained by averaging distributions from the sidebands $\mathbf{A}$ and $\mathbf{B}$. The estimated background distribution is subtracted from the 


\begin{tabular}{|c|c|c|c|c|}
\hline \multirow[b]{2}{*}{ sideband subtraction } & \multicolumn{2}{|c|}{ SUSY point A1 } & \multicolumn{2}{|c|}{$t \bar{t}$} \\
\hline & before & after & before & after \\
\hline No lepton cut & 59174 & 13340 & $\begin{array}{r}8168 \\
(0.138)\end{array}$ & $\begin{array}{r}2764 \\
(0.207)\end{array}$ \\
\hline Loose lepton cut & 47171 & 10487 & $\begin{array}{r}5789 \\
(0.123)\end{array}$ & $\begin{array}{r}1777 \\
(0.169)\end{array}$ \\
\hline Hard lepton cut & 26915 & 6114 & $\begin{array}{r}2671 \\
(0.099) \\
\end{array}$ & $\begin{array}{r}884 \\
(0.145) \\
\end{array}$ \\
\hline
\end{tabular}

TABLE II: Numbers of events having a top candidate with or without the lepton cuts. The integrated luminosity is $120 \mathrm{fb}^{-1}$. The numbers in parentheses are the ratios of the $t \bar{t}$ events to the SUSY events.
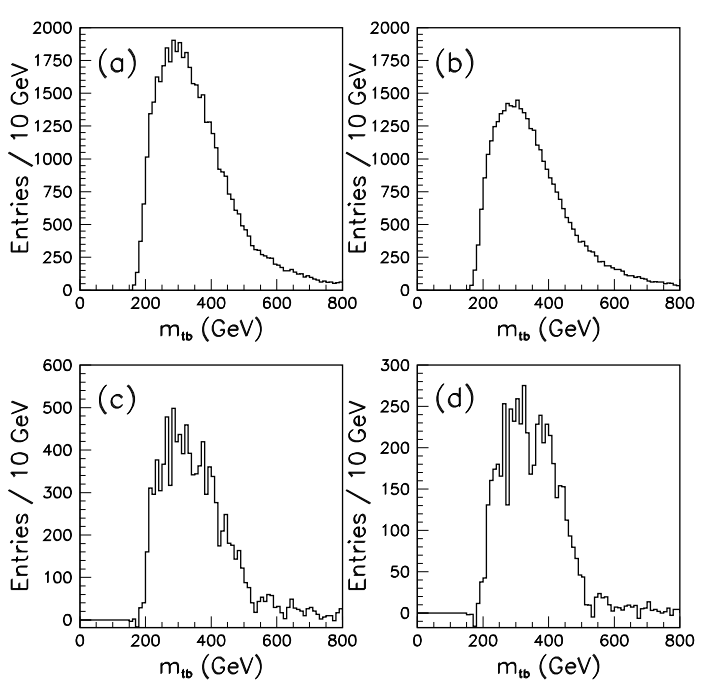

FIG. 6: (a) Signal $m_{t b}$ distribution for the sample point A1 in Table I, (b) the estimated background distribution from the sideband events, (c) (a) - (b), and (d) the $m_{t b}$ distribution for the modes (III) $)_{1}$ and (IV) 11 in Eq. (1), and the decay modes $(\text { III })_{i 1}$ irreducible to the mode (III $)_{1}$.

signal distribution in Fig. 6(c). The corrected signal distribution (c) shows a better end point structure compared to (a).

By using the information from the event generator, one can show the sideband method is indeed reproduces the $m_{t b}$ distribution for the signal modes (III) and (IV). Fig. 6(d) is the same distribution as Fig. 6(c) but for the events which contain either the decay chain (III) $)_{1}$, the decay chain (IV) $)_{11}$, or the decay chains (III) ${ }_{i 1}$, which are irreducible to the chain (III) $)_{1} ; \tilde{g} \rightarrow b \tilde{b}_{i} \rightarrow b W \tilde{t}_{1} \rightarrow b b W \tilde{\chi}_{1}^{ \pm}$. Note that if $b W$ has an invariant mass consistent to a top quark, the decay is kinematically equivalent to the mode (III) ${ }_{1}$. Fig. 6 (d) shows two end points as expected $\left(M_{t b}(\mathrm{III})_{1}=471 \mathrm{GeV}\right.$ and $\left.M_{t b}(\mathrm{IV})_{11}=420 \mathrm{GeV}\right)$, demonstrating that the sideband method works well.

Note that the signals from the modes (III) and (IV) in Eq. (1) are significant in the total selected events. The total distribution shown in Fig. 6(c) contains contribution from mis-reconstructed events such as other gluino decay chains with $W$ and $b$-jets, or stop/sbottom pair productions.

We fit the total distribution shown in Fig. 6(c), which is made with a bin size $\Delta m=10 \mathrm{GeV}$, by a simple fitting function described with the end point $M_{t b}^{\mathrm{fit}}$, the edge height $h$ per $\Delta m$ bin, and the smearing parameter $\sigma$ originated from the jet energy resolution;

$f\left(m_{t b}\right)=\frac{h}{M_{t b}^{\mathrm{fit}}} \int_{m_{t}+m_{b}}^{M_{t b}^{\mathrm{fit}}} \frac{m}{\sqrt{2 \pi} \sigma} \exp \left(-\frac{1}{2}\left[\frac{m-m_{t b}}{\sigma}\right]^{2}\right) d m$.

To reduce the number of free parameters for good convergence capability of the fit, we set the smearing parameter $\sigma$ to be $10 \%$ of the end point $M_{t b}^{\mathrm{fit}}$. This assumption is based on the dijet mass resolution of the ATLAS detector [2]. We assume that the signal distribution is sitting on a linearly-decreasing background expressed by a function $a\left(m_{t b}-M_{t b}^{\mathrm{fit}}\right)+b$. The parameters $a$ and $b$ are also determined by the fit.

The fit results, especially the edge height $h$, depend on the mass range used for the fit. We therefore apply an iterative fitting procedure to obtain stable results. The initial $M_{t b}^{\text {fit }}$ value is determined by fitting a rather wide mass range $350 \mathrm{GeV}<m_{t b}<800 \mathrm{GeV}$ (45 histogram bins). In the next step, lower 8 bins and higher 25 bins with respect to the $M_{t b}^{\text {fit }}$ value are used to obtain new fit results including the new $M_{t b}^{\text {fit }}$ value. The relatively small number of lower bins is chosen because the edge height is sensitive to the distribution in the mass region near the end point. Including too many lower bins is found to degrade the sensitivity. The higher bins determines the linearly-decreasing background. This step is repeated until the fit results become stable. The number of iterations is typically three or four. If the edge height $h$ has a large fit error, the number of lower bins is increased to 9 or 10 , until the error becomes reasonably small (typically less than $15 \%$ ). The fit result is shown in Fig. 7. We obtain $M_{t b}^{\mathrm{fit}}=455.2 \pm 8.2 \mathrm{GeV}$ and $h=271 \pm 23 /(10 \mathrm{GeV})$, where the errors are statistical.

\section{THE $m_{t b}$ DISTRIBUTIONS IN THE SUSY MODEL POINTS}

In the previous section we discuss the selection of the $t b$ events and the extraction of the edge structure in the $m_{t b}$ distribution originated from the gluino decays (III) $)_{1}$, $(\mathrm{III})_{i 1}$ or $(\mathrm{IV})_{i 1}$. Two values are obtained from the $m_{t b}$ distribution; the end point $M_{t b}^{\mathrm{fit}}$ is directly related to the stop, sbottom, and gluino masses, while the edge height $h$ is sensitive to the gluino production cross section and the decay branching ratios.

This section is aimed to compare the reconstructed values with expectations. We will see that the kinematical quantities $M_{t b}^{\mathrm{fit}}$ and the peak value of the effective mass 


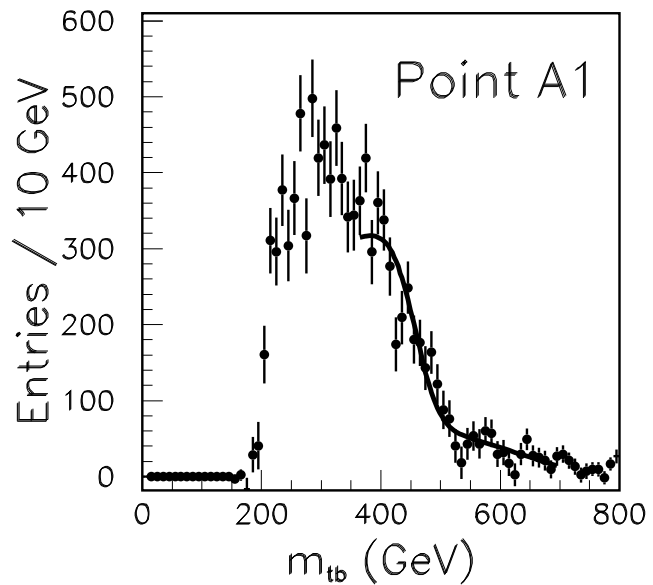

FIG. 7: A fit to the $m_{t b}$ distribution at the point A1.

distributions $\left(M_{\text {eff }}\right)$ agree very well to $M_{t b}^{\mathrm{w}}$ and $m_{\tilde{q}}+m_{\tilde{g}}$, respectively. On the other hand, the reconstructed edge height $h$, which is related to the total number of gluinos decaying through the modes (III) or (IV), is dependent on the event generator, and also on the decay patterns of the neutralino, chargino and squarks. We clarify key issues to reduce the uncertainties in this section.

\section{A. Model parameters}

The gluino decay widths and the decay kinematics depend on the squark masses and mixings. The charginos and neutralinos in the decay chains (I)-(IV) further decay into jets/leptons, and the decay patterns depend on the electroweak SUSY parameters such as $\mu, M_{1}, M_{2}, \tan \beta$ and the slepton masses. The $m_{t b}$ distribution is therefore a function of all SUSY parameters. To check the validity of our reconstruction and subtraction scheme, we study the $m_{t b}$ distributions for various sets of SUSY parameters.

The reference point A1 introduced in the previous section corresponds to the MSUGRA model with a large negative $A_{0}$ value. The stop and sbottom masses can be changed by varying the $A_{0}$ value without changing other sparticle masses drastically (see Section V for the detail). We thus make another MSUGRA point A2, by changing only the $A_{0}$ value from $-300 \mathrm{GeV}$ to $300 \mathrm{GeV}$ from the point $\mathrm{A} 1$. In addition, two non-MSUGRA points $\mathrm{T} 1$ and $\mathrm{T} 2$ are made from the point $\mathrm{A} 1$, where the chargino/neutralino sector and the gluino mass are kept unchanged but the stop masses and mixing are modified by changing the mass parameter $m_{\tilde{t}_{R}}$. The stop mass parameters and the mixing angle at the low energy scale are listed in Table III.

\begin{tabular}{|c||c|c|c|c|c|}
\hline & $m_{\tilde{t}_{1}}$ & $m_{\tilde{t}_{R}}$ & $m_{\tilde{t}_{L}}$ & $A_{t}$ & $\theta_{\tilde{t}}$ \\
\hline $\mathrm{A} 1$ & 427 & 482 & 573 & -655 & 0.99 \\
$\mathrm{~A} 2$ & 496 & 521 & 591 & -457 & 1.01 \\
$\mathrm{~T} 1$ & 327 & 366 & 573 & -655 & 1.14 \\
$\mathrm{~T} 2$ & 477 & 551 & 573 & -655 & 0.84 \\
\hline
\end{tabular}

TABLE III: The lighter stop mass and the relevant SUSY mass parameters in $\mathrm{GeV}$ and the stop mixing angles for the points A1, A2, T1 and T2.

\begin{tabular}{|c||c|c|c|c|c|}
\hline & $M_{0}$ & $m_{0}(\tilde{q})$ & $m_{0}(H)$ & $A_{0}$ & $\tan \beta$ \\
\hline \hline A1 & 300 & 100 & 100 & -300 & 10 \\
A2 & 300 & 100 & 100 & 300 & 10 \\
\hline B & 255 & 102 & 102 & 0 & 10 \\
C & 408 & 92 & 92 & 0 & 10 \\
G & 383 & 125 & 125 & 0 & 20 \\
I & 358 & 188 & 188 & 0 & 35 \\
\hline E1 & 200 & 500 & 200 & -1000 & 10 \\
E1 & 300 & 700 & 500 & -1000 & 10 \\
\hline
\end{tabular}

TABLE IV: The universal parameters at the GUT scale for the points we study. Units are in $\mathrm{GeV}$ except $\tan \beta$.

Next we choose MSUGRA points B, C, G and I, which were discussed in the paper [15]. They satisfy $m_{\tilde{g}}>m_{\tilde{t}_{1}}+$ $m_{t}$ so that the decay mode (III) $)_{1}$ is open. The points also satisfy the condition $m_{\tilde{g}}<1 \mathrm{TeV}$, which is required for a statistical reason. The gluino production is dominated by the process $q g \rightarrow \tilde{q} \tilde{g}$ in the case of $m_{\tilde{q}} \sim m_{\tilde{g}}$, and the production cross section is parameterized as [16]

$$
\sigma(\tilde{q} \tilde{g})+\sigma\left(\tilde{q}^{*} \tilde{g}\right)=1.74 \times\left(\frac{m_{\tilde{g}}}{\mathrm{TeV}}\right)^{-5.78}(\mathrm{pb}) .
$$

For example the cross section is $14 \mathrm{pb}$ and $3.1 \mathrm{pb}$ for $m_{\tilde{g}}=700 \mathrm{GeV}$ and $900 \mathrm{GeV}$, respectively. Assuming that the branching ratios and the reconstruction efficiency are equal to those of the point $\mathrm{A} 1$, the gluino mass must be less than $1 \mathrm{TeV}$ to reconstruct more than 2000 SUSY $t b$ events after the sideband subtraction for an integrated luminosity of $100 \mathrm{fb}^{-1}$.

Finally we select two more points E1 and E2, where only the decay chain (III) ${ }_{1}$ is kinematically open and the other two body gluino decays into squarks are closed. This happens when $m_{0}$ and $A_{0}$ are large. As the SUSY events at these points typically contain four bottom quarks, the combinatorial background for the $t b$ reconstruction is significantly large.

For the points we study in this paper, we list the relevant sparticle masses and the universal parameters at the GUT scale in Table I and Table IV, respectively. At each SUSY point we generate two Monte Carlo data samples, each having $3 \times 10^{6}$ events, where one is generated by PYTHIA and the other by HERWIG. The cross sections are summarized in Table I. The SUSY cross section for the point $\mathrm{C}$ is the smallest among them, where the gener- 
ated $3 \times 10^{6}$ events correspond to an integrated luminosity of $600 \mathrm{fb}^{-1}$.

\section{B. Reconstruction of kinematic variables}

The fit results of the $m_{t b}$ distributions are listed in Table V, where we follow the fitting procedure described in the previous section. The weighted end point $M_{t b}^{\mathrm{w}}$ is defined in Eq. (3) and the relevant branching ratios are listed in Table VI. In Table V, $N_{\text {all }}$ is the number of $t b$ events after the sideband subtraction, while $N_{\text {edge }}$ is the number of $t b$ events after the subtraction, with one and only one gluino decaying through the mode $(\mathrm{III})_{1},(\mathrm{III})_{i 1}$ or $(\mathrm{IV})_{i 1}$. Generator information is used to obtain $N_{\text {edge }}$.

The relation between $M_{t b}^{\mathrm{fit}}$ and $M_{t b}^{\mathrm{w}}$ is shown in

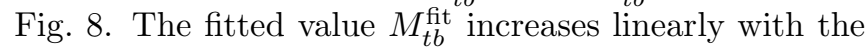
weighted end point $M_{t b}^{\mathrm{w}}$ in the mass range $350 \mathrm{GeV}<$ $M_{t b}^{\mathrm{w}}<600 \mathrm{GeV}$, and tends to be lower than $M_{t b}^{\mathrm{w}}$. In the previous literature $[2,9]$, the lower mass value was understood as the effect of particles missed outside the jet cones. In the literature invariant mass distributions of the events with same flavor and opposite sign leptons are studied, which comes from the cascade decay of the squarks $\tilde{q} \rightarrow q \tilde{\chi}_{2}^{0} \rightarrow q l \tilde{l} \rightarrow q l l \tilde{\chi}_{1}^{0}$. The end point of the $m_{j l l}$ distribution is lower than the parton level qll end point by about $10 \%$.

In the study of the squark cascade decay $\tilde{q} \rightarrow q l l \tilde{\chi}_{1}^{0}$, the end point agrees better with the parton level one by changing the jet cone size to $R=0.7$. In our study at the reference point $\mathrm{A} 1$, the reconstructed number of events is significantly reduced for $R=0.7$. We find that using the jet cone size $R=0.5$ leads to a better reconstruction and a larger $M_{t b}^{\mathrm{fit}}$, which is closer to $M_{t b}^{\mathrm{w}}$. Such comparison might be useful to estimate the true end point. We discuss the dependence on the jet finding algorithm and the cone parameters in Appendix.

We note that our definition of $M_{t b}^{\mathrm{w}}$ might be too simple if the reconstruction efficiencies of the decay modes (III) and (IV) are very different. In addition, the weighted average should not be applied when $M_{t b}(\mathrm{III})_{1}$ and $M_{t b}(\mathrm{IV})_{11}$ differ by more than $80 \mathrm{GeV}$, as we use the events with $m_{t b}>M_{t b}^{\text {fit }}-80 \mathrm{GeV}$ for the fit.

We now discuss the relation between $M_{\text {eff }}$ and $m_{\tilde{g}}, m_{\tilde{q}}$ using the $t b$ samples. We find that sum of the masses $m_{\tilde{g}}+m_{\tilde{q}}$ has a linear dependence on $M_{\text {eff }}$ as shown in Fig. 9. The deviation of the sample points from a linear fit is less than 5\%. The plot is for HERWIG samples and we find that PYTHIA and HERWIG give consistent results for the relation between $M_{\text {eff }}$ and the masses.

The $t b$ sample contains two and only two $b$-jets originating from a gluino decay, therefore the $m_{\tilde{g}}$ dependence of $M_{\text {eff }}$ is expected. One may wonder that the $\tilde{g} \tilde{g}$ production might affect the $M_{\text {eff }}$ value. This is, however, not the case for the points we study. Indeed, the points E1 and E2, where $m_{\tilde{q}} \gg m_{\tilde{g}}$, also satisfy the linear relation between $M_{\text {eff }}$ and $m_{\tilde{q}}+m_{\tilde{g}}$. Note that the quark parton distribution is harder than the gluon parton distribution.

\begin{tabular}{|c||cccc|cc|}
\hline & gen & $M_{t b}^{\mathrm{w}}[\mathrm{GeV}]$ & $M_{t b}^{\mathrm{fit}}[\mathrm{GeV}]$ & $h /(10 \mathrm{GeV})$ & $N_{\text {edge }}$ & $N_{\text {all }}$ \\
\hline A1 & PY & 459 & $455.2 \pm 8.3$ & $271.4 \pm 22.7$ & 5846 & 10487 \\
& HW & & $434.5 \pm 5.8$ & $354.8 \pm 23.3$ & 6685 & 11470 \\
\hline A2 & PY & 409 & $442.0 \pm 17.5$ & $153.0 \pm 20.6$ & 3064 & 7525 \\
& HW & & $394.4 \pm 9.5$ & $190.6 \pm 21.8$ & 3095 & 7805 \\
\hline T1 & PY & 468 & $460.3 \pm 5.4$ & $327.0 \pm 21.6$ & 6620 & 11659 \\
& HW & & $452.0 \pm 3.9$ & $447.5 \pm 23.5$ & 8170 & 14050 \\
\hline T2 & PY & 429 & $434.5 \pm 8.1$ & $223.2 \pm 21.6$ & 4461 & 8466 \\
& HW & & $416.6 \pm 5.2$ & $321.0 \pm 23.2$ & 5378 & 9592 \\
\hline B & PY & 371 & $385.6 \pm 6.3$ & $226.9 \pm 19.6$ & 2801 & 5396 \\
& HW & & $361.7 \pm 7.3$ & $223.5 \pm 21.3$ & 3105 & 5935 \\
\hline C & PY & 557 & $548.2 \pm 14.1$ & $142.4 \pm 17.5$ & 4026 & 11228 \\
& HW & & $556.3 \pm 9.4$ & $178.2 \pm 17.9$ & 4395 & 12704 \\
\hline G & PY & 533 & $498.5 \pm 8.6$ & $244.1 \pm 23.8$ & 5784 & 13630 \\
& HW & & $506.9 \pm 6.4$ & $325.5 \pm 22.9$ & 6248 & 15039 \\
\hline I & PY & 507 & $497.8 \pm 7.3$ & $289.7 \pm 24.0$ & 6016 & 13752 \\
& HW & & $492.9 \pm 5.2$ & $383.9 \pm 24.5$ & 6661 & 14968 \\
\hline E1 & PY & 360 & $345.5 \pm 5.3$ & $270.6 \pm 23.7$ & 2778 & 4595 \\
& HW & & $348.0 \pm 6.3$ & $251.5 \pm 23.9$ & 3169 & 5167 \\
\hline E2 & PY & 453 & $430.8 \pm 7.5$ & $352.0 \pm 33.9$ & 5577 & 16490 \\
& HW & & $444.7 \pm 8.0$ & $324.6 \pm 30.9$ & 4394 & 15724 \\
\hline
\end{tabular}

TABLE V: Fit results of the $m_{t b}$ distributions and numbers of $t b$ events after the sideband subtraction $N_{\text {edge }}$ and $N_{\text {all }}$ for $3 \times 10^{6}$ Monte Carlo events. The fit does not include the $t \bar{t}$ background, and the loose lepton cut is applied. $N_{\text {all }}$ is the total number of $t b$ events, while $N_{\text {edge }}$ is the number of events with one and only one gluino decays into mode (III) ${ }_{1},(\text { III })_{i 1}$ or $(\mathrm{IV})_{i 1}$. "PY" is for PYTHIA and "HW" is for HERWIG.

Therefore $\sigma(p p \rightarrow \tilde{q} \tilde{g}) \gg \sigma(p p \rightarrow \tilde{g} \tilde{g})$ in a wide MSSM parameter region. This is why $M_{\text {eff }}$ becomes a very good function of $m_{\tilde{g}}+m_{\tilde{q}}$.

In Ref. [17] the relation between $M_{\text {eff }}$ and the effective SUSY scale is studied, where the effective SUSY scale is defined as the cross section weighted mean of the masses of two sparticles initially produced in $p p$ collisions. On the other hand, we actively select the $\tilde{g} \tilde{q}$ productions by requiring two tagged $b$-jets. This leads to a clear dependence of $M_{\text {eff }}$ and $m_{\tilde{q}}+m_{\tilde{g}}$.

\section{Number of $t b$ events}

We now discuss the relation between the edge height $h$, the number of reconstructed $t b$ events, and the reconstruction efficiencies. The total number of the "edge" events $N_{\text {edge }}$ arising from the decay chains (III) and (IV) may be estimated from $M_{t b}^{\mathrm{fit}}, h$ per the bin size $\Delta m$ as follows,

$$
N_{\text {edge }} \sim N_{\text {fit }}=\frac{h}{2}\left(\frac{m_{t}}{M_{t b}^{\mathrm{fit}}}+1\right) \times \frac{M_{t b}^{\mathrm{fit}}-m_{t}}{\Delta m} .
$$

This formula is obtained by assuming the parton level distribution, and equating the minimum of the $m_{t b}$ dis- 


\begin{tabular}{|c||c|c|c|c|c|c|c|}
\hline & $(\mathrm{III})_{1}$ & $(\mathrm{IV})_{11}$ & $(\mathrm{IV})_{21}$ & $(\mathrm{III})_{11}$ & $(\mathrm{III})_{21}$ & sum & $b b X$ \\
\hline $\mathrm{A} 1$ & 11.0 & 6.7 & 1.4 & 3.4 & 2.7 & 25.3 & 43.4 \\
$\mathrm{~A} 2$ & 3.1 & 6.5 & 1.6 & 1.4 & 0.4 & 13.1 & 32.0 \\
$\mathrm{~T} 1$ & 24.5 & 3.2 & 0.8 & 5.0 & 3.0 & 36.5 & 56.3 \\
$\mathrm{~T} 2$ & 4.3 & 9.9 & 2.2 & 0.5 & 2.1 & 19.0 & 36.2 \\
$\mathrm{~B}$ & 4.1 & 8.2 & 2.3 & 0.9 & 1.7 & 17.3 & 33.5 \\
$\mathrm{C}$ & 7.2 & 5.3 & 1.3 & 0.9 & 0.8 & 15.4 & 38.5 \\
$\mathrm{G}$ & 6.6 & 7.5 & 1.2 & 0.5 & 0.8 & 16.6 & 40.4 \\
$\mathrm{I}$ & 6.2 & 11.1 & 0.7 & 0.0 & 0.7 & 18.7 & 47.3 \\
$\mathrm{E} 1$ & 78.5 & 0 & 0 & 0 & 0 & 78.5 & 99 \\
$\mathrm{E} 2$ & 42.6 & 0 & 0 & 0 & 0 & 42.6 & 98 \\
\hline
\end{tabular}

TABLE VI: Branching ratios of gluino cascade decays in $\%$. The decay modes (III) and (IV) are defined in Eq. (1). The "sum" is total of all (III) $)_{1},(\text { III })_{i 1}$ and (IV) $)_{i 1}$ decay modes. The " $b b X$ " is the branching ratio of the gluino decaying into $\tilde{t}_{i}$ or $\tilde{b}_{i}$, so that two bottom quarks appear in the decay products.

For $3 \times 10^{6}$ SUSY events

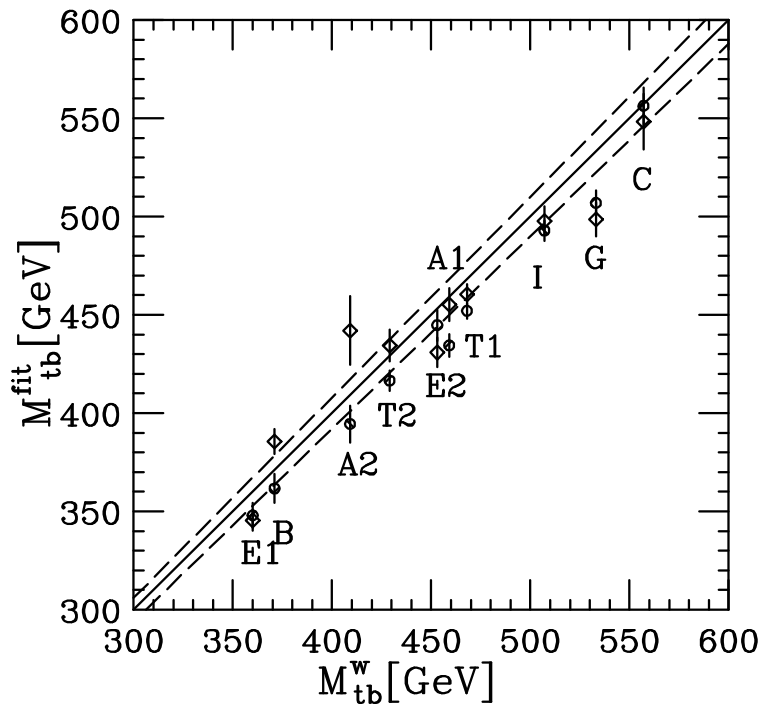

FIG. 8: Relation between $M_{t b}^{\mathrm{w}}$ and $M_{t b}^{\mathrm{fit}}$ for the sample points. The solid line corresponds to $M_{t b}^{\mathrm{w}}=M_{t b}^{\mathrm{fit}}$ and dashed lines to $M_{t b}^{\mathrm{w}}(1 \pm 0.02)=M_{t b}^{\mathrm{fit}}$. Bars with a diamond and a circle correspond to PYTHIA and HERWIG samples, respectively.

tribution from the decay chain (III) or (IV) to $m_{t}$. This is a good approximation for reasonable SUSY parameters.

In Fig. 10(a) and (b) we compare $N_{\text {edge }}$ in Table V with $N_{\text {fit }}$. We find a very good agreement between them both for the PYTHIA and HERWIG samples. On the other hand, the correlation between $N_{\text {all }}$ and $N_{\text {fit }}$ is much worse as shown in Fig. 10(c). The number $N_{\text {all }}$ receives contribution from other gluino cascade decay chains such as the modes (I) and (II), as well as contributions from the stop and the sbottom pair productions. By the end point fit, we extract number of events coming from only the modes (III) $)_{1}$, (III) $)_{i 1}$ and (IV) $)_{i 1}$.

$N_{\text {edge }}$, and then $N_{\text {fit }}$, must be related to the number of

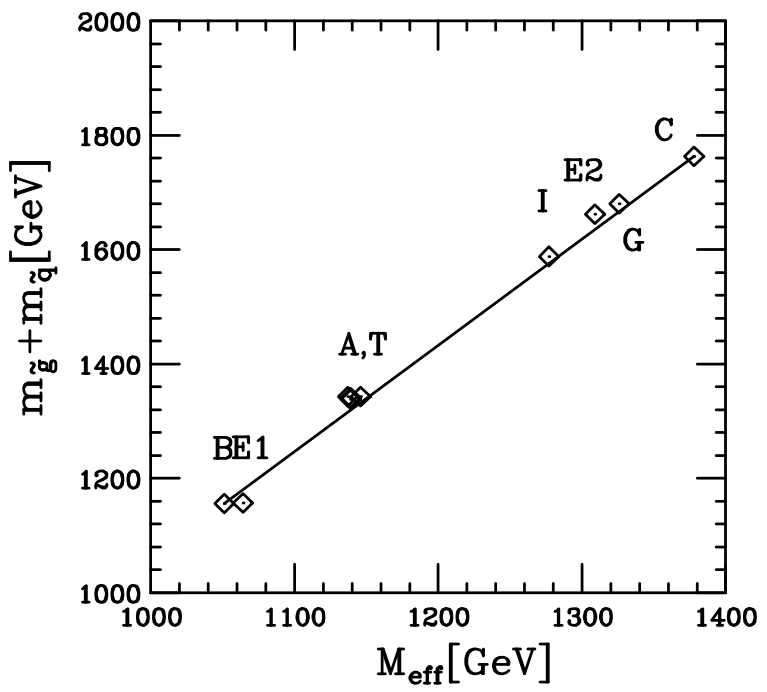

FIG. 9: Relation between $M_{\text {eff }}$ and $m_{\tilde{g}}+m_{\tilde{q}}$ for the sample points in Table I (HERWIG samples). The line shows a linear fit of $m_{\tilde{g}}+m_{\tilde{q}}$ as a function of $M_{\text {eff }}$.

gluino decays through the cascade decay chains (III) and (IV) via the reconstruction efficiencies. For the points we study, the number of produced $\tilde{g} \tilde{g}$ events, $N(\tilde{g} \tilde{g})$, is typically $10 \%$ to $14 \%$ of the total SUSY production events, while the number of $\tilde{q}^{*} \tilde{g}$ and $\tilde{q} \tilde{g}$ production events $N\left(\tilde{q}^{*} \tilde{g}\right)+N(\tilde{q} \tilde{g})$ ranges from $42 \%$ to $51 \%$. The gluino decay branchings ratios are listed in Table VI. The number of events $N_{\text {prod }}$, where one gluino decays through the modes (III) or (IV) and the other squark or gluino decay does not produce any bottom quark, is given as follows;

$$
\begin{aligned}
N_{\text {prod }}= & 2 N(\tilde{g} \tilde{g})(1-\operatorname{Br}(\tilde{g} \rightarrow b b X)) \operatorname{Br}(\text { edge }) \\
& +\left(N(\tilde{g} \tilde{q})+N\left(\tilde{g} \tilde{q}^{*}\right)\right) \operatorname{Br}(\text { edge }) \\
\operatorname{Br}(\text { edge }) \equiv & \operatorname{Br}(\mathrm{III})_{1}+\operatorname{Br}(\mathrm{III})_{11}+\operatorname{Br}(\mathrm{III})_{21} \\
& +\operatorname{Br}(\mathrm{IV})_{11}
\end{aligned}
$$

where $\operatorname{Br}(\tilde{g} \rightarrow b b X)$ is the branching ratio of the gluino decaying into stop or sbottom, thus having two bottom quarks in the final state. The reconstruction efficiency of the $t b$ edge mode, $\epsilon_{t b}$, is given as

$$
\epsilon_{t b}=N_{\text {edge }} / N_{\text {prod }}
$$

If the efficiency does not strongly depend on uncertainty in hadronization and the model parameter dependence can be corrected from other measurements, we can extract the number $N_{\text {prod }}$ from the experimental data.

The major uncertainty in hadronization may be estimated by the generator dependence of the reconstruction efficiency $\epsilon_{t b}$. The edge height $h$ for the HERWIG sample is significantly larger than that of the PYTHIA sample in Table V, except the points E1 and E2, and the difference is more than $20 \%$ at many points. We note the difference is small before we apply the sideband subtraction. For example, the numbers of $t b$ events before and after the sideband subtraction are 9695 (10180) and 

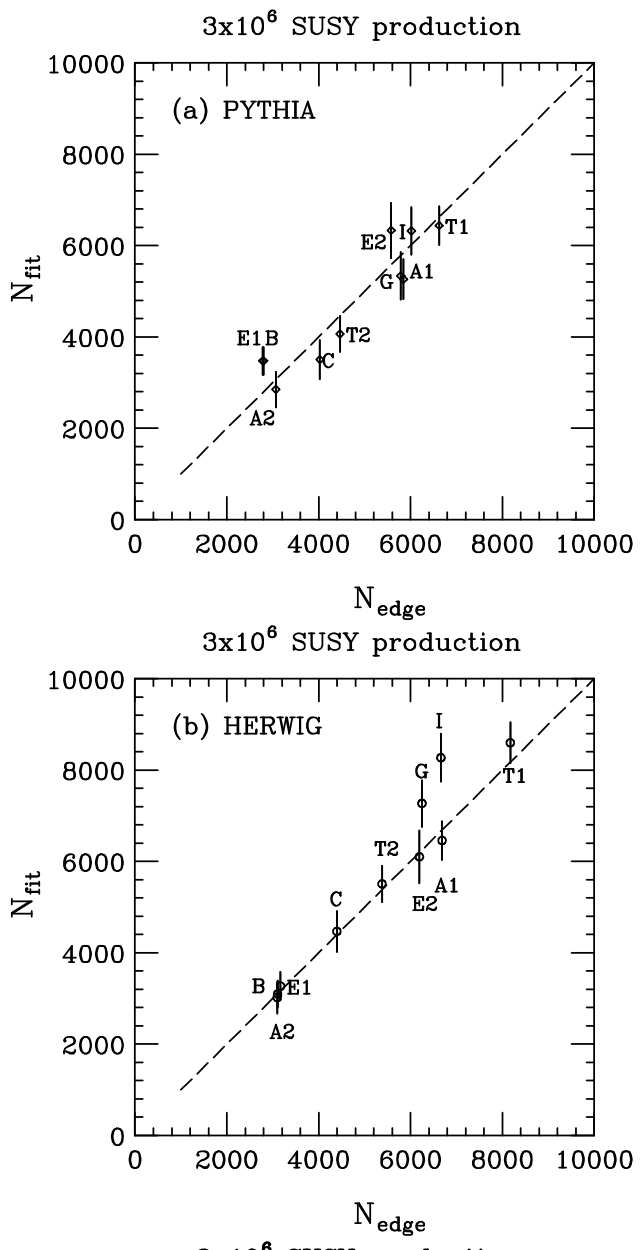

for the HERWIG sample. Indeed, the major difference of the two generators are in the fragmentation scheme. PYTHIA is based on the string model, while HERWIG is based on the QCD model. Currently we are only able to say that understanding the nature of fragmentation is essential for the interpretation of $N_{\text {fit }}$. We compare the HERWIG and PYTHIA samples in Appendix.

Let us discuss other effects that might change the reconstruction efficiency. In Fig. 11 we plot the reconstruction efficiency $\epsilon_{t b}$ as a function of $m_{\tilde{g}}$ by normalizing the efficiency to that for the point A1. Here $N_{\text {prod }}$ is calculated using Eq. (7), the generator information on $N(\tilde{g} \tilde{g}), N\left(\tilde{q}^{(*)} \tilde{g}\right)$, and the Monte Carlo inputs of the gluino branching ratios. The length of the bar in the plot shows twice of the statistical error of $N_{\text {edge }}$, $\delta\left(\epsilon_{t b} / \epsilon_{t b}(A 1)\right)=2\left(\epsilon_{t b} / \epsilon_{t b}(\mathrm{~A} 1)\right) / \sqrt{N_{\text {edge }}}$. We don't discuss the points E1 and E2 here because events with two bottom quarks are not the dominant signature of squark and gluino production at these points.

The efficiencies are very close to one another among the points A1, A2, T1, and T1. Although the stop mass is very different among the points, the mass has little influence on the efficiency. Note that these points have the same gluino masses, and almost the same parameters for the chargino and neutralino sector. For the other points, the efficiency can also be expressed as a linear function of $m_{\tilde{g}}$ except the sample point C. The gluino mass dependence arises from the pre-selection cut $m_{\mathrm{eff}}>$ $1000 \mathrm{GeV}$.

The reconstruction efficiency at the point $\mathrm{C}$ (a circle in Fig. 11) is lower by $20 \%$ from the linear fit (the dashed line in Fig. 11). This is due to the loose lepton cut which reduces the $t \bar{t}$ background. As the chargino decay branching ratio into leptons is high (about 47\%) at the point $\mathrm{C}$, the lepton cut kills a significant fraction of $t b$ events. The effect is estimated by comparing $N_{\text {edge }}$ without any lepton cut and $N_{\text {edge }}$ with the loose lepton cut, at the points $\mathrm{C}$ and $\mathrm{A} 1$. The efficiency at the point $\mathrm{C}$ without any lepton cut is plotted in Fig. 11, and is close to the linear fit of the other points.

The gluino mass dependence of the reconstruction efficiency would be easily corrected by estimating the gluino mass from the effective mass distribution. The uncertainty from the chargino decay branching ratio may be corrected by studying $t b$ final states with leptons as well. Therefore the parameter dependence of the efficiency may be corrected by analyzing the Monte Carlo and real

FIG. 10: Relations between $N_{\text {edge }}$ and $N_{\text {fit }}$ for (a) PYTHIA and (b) HERWIG. (c) Relation between $N_{\text {all }}$ and $N_{\text {fit }}$. The line in (a) and (b) shows $N_{\text {fit }}=N_{\text {edge }}$, while the line in (c) is a result of linear fit.

2462 (2949) for the PYTHIA (HERWIG) sample, respectively, at the point A1. The number of events before the sideband subtraction only differs by $5 \%$ between HERWIG and PYTHIA, while the difference increases to be more than $20 \%$ after the subtraction. This indicates that the resolution of the $W$ boson mass is somewhat better data. Efforts to determine the MSSM parameters are of course very important to this purpose.

\section{Branching ratios}

In the previous subsection, we find that the reconstruction efficiencies significantly differ between the PYTHIA and HERWIG samples. However, as we can see in Table $\mathrm{V}$, the generator dependence mostly cancels in the ratio $N_{\text {fit }} / N_{\text {all }}$. Therefore the ratio may play a role to 
acceptance vs gluino mass

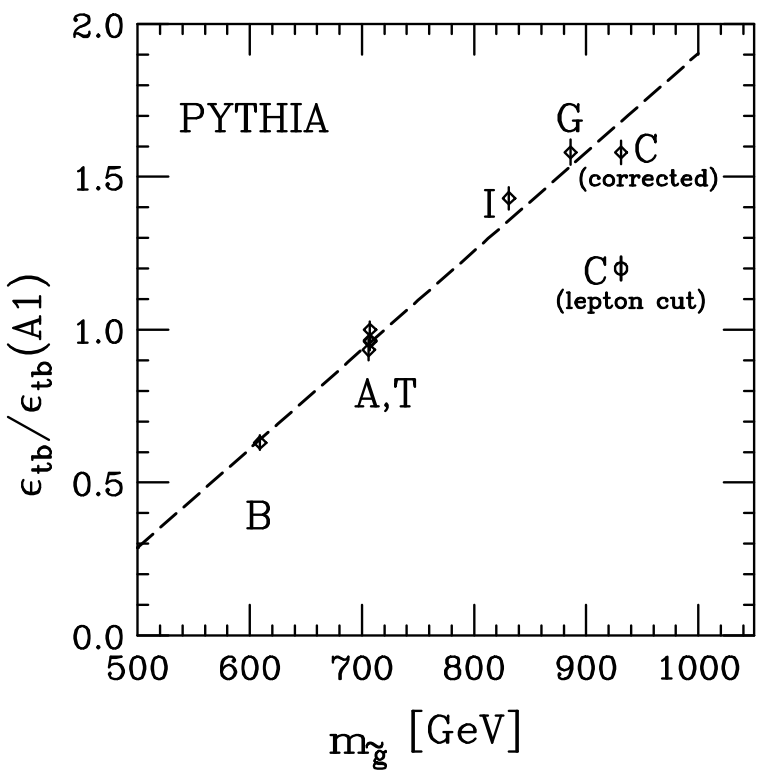

FIG. 11: Reconstruction efficiencies of the model points in Table I relative to that of the point $\mathrm{A} 1$ as a function of $m_{\tilde{g}}$. Point $\mathrm{C}$ is plotted twice with/without the loose lepton cut.

determine the fundamental parameters. For example, when the contributions from the stop or sbottom pair productions are negligible, $N_{\text {fit }} / N_{\text {all }}$ is determined by the branching ratios to the third generation squarks. However, there are several aspects one must consider.

$N_{\text {all }}$ consists of contributions from various decay modes, even if the contributions from the stop or sbottom pair productions are negligible:

- The $t \bar{t}$ final state from the decay chains (II) contributes to $N_{\text {all }}$. The branching ratio is typically $1 / 3$ of that of the $t b$ final state for $\mu>M_{2}>M_{1}$. The $t \bar{t}$ final state is reconstructed as $t b$ with a high probability, although the $m_{t b}$ distribution does not have an edge structure.

- We expect that events having no $t \rightarrow b W$ decay would be eliminated by the sideband subtraction, and should not contribute to $N_{\text {all }}$. However, some events actually remain, because of $W$ and $Z^{0}$ bosons from the decay of charginos and neutralinos, as well as accidental $W$ and $Z^{0}$ bosons from other cascade decay chains. An example where $\tilde{\chi}_{2}^{0}$ decays dominantly into the $Z^{0}$ boson will be discussed in the next subsection.

In addition, the sideband subtraction is not necessarily perfect, as will be discussed in Appendix. For example, the efficiency of the mode $(\mathrm{I})_{2}$ is roughly a half of that of the mode (III) ${ }_{1}$ after the sideband subtraction at the point A1 (see Appendix).

- The cuts to reduce background events may induce decay mode dependence of the efficiency. For ex- ample the lepton cut could efficiently reduce the edge mode (III) if $\operatorname{Br}\left(\tilde{\chi}_{1}^{ \pm} \rightarrow l X\right)$ is large as we have seen in Fig. 11 for point $C$.

The interpretation of the ratio $N_{\text {fit }} / N_{\text {all }}$ becomes more complicated for the following two cases:

- If $m_{\tilde{t}(\tilde{b})}<m_{\tilde{g}}<m_{\tilde{q}}$, squarks mostly decay into the gluino. As discussed previously, the squark/gluino production and decay typically produce events with four $b$-jets. They are identified as a two $b$-jet event when two $b$-jets are tagged and the other two are mis-tagged. These events decrease the fraction from the decay chain (III) and (IV) in the two $b$-jet events. Assuming a tagging efficiency of $60 \%$ for a single $b$-jet, $35 \%$ of the four bottom quark events are identified as two $b$-jet events, and only $1 / 3$ of them contain a $b$-jet pair originated from a single gluino decay. The wrong $b$-jet pair combinations significantly dilute the edge mode. Study of events with three $b$-jets and four $b$-jets is necessary for this case.

- The $t b$ signal from the stop pair production could be significant if the stop is much lighter than the gluino. For example, only $4.7 \%$ of the SUSY events comes from the stop pair production at the point A1, while it amounts to be about $17 \%$ at the point T1.

In the MSUGRA model, both the right-handed stop and the left-handed sbottom is lighter than gluino in a broad parameter space. As a result, the decay modes which involve $W$ bosons (modes (II), (III) and (IV)) dominate over the gluino decays to $b b X$ in the region. Because the events with $W$ bosons remain after the $W$ sideband subtraction, the reconstruction efficiency $\epsilon_{t b}$ is expected to be similar for those decay modes. Thus, if the contributions from the stop or sbottom pair productions are negligible, the numbers of events with two bottom quarks are given approximately as

$$
\begin{aligned}
& N_{\text {fit }} \sim \epsilon_{t b} \operatorname{Br}(\text { edge }) \times \\
& \quad\left[2 N(\tilde{g} \tilde{g})(1-\operatorname{Br}(\tilde{g} \rightarrow b b X))+N(\tilde{g} \tilde{q})+N\left(\tilde{g} \tilde{q}^{*}\right)\right], \\
& N_{\text {all }} \sim \epsilon_{t b} \operatorname{Br}(\tilde{g} \rightarrow b b X) \times \\
& \quad\left[2 N(\tilde{g} \tilde{g})(1-\operatorname{Br}(\tilde{g} \rightarrow b b X))+N(\tilde{g} \tilde{q})+N\left(\tilde{g} \tilde{q}^{*}\right)\right] .
\end{aligned}
$$

If this simple formula holds, the ratio $N_{\text {fit }} / N_{\text {all }}$ should provide the ratio of the branching ratios $\operatorname{Br}($ edge $) / \operatorname{Br}(\tilde{g} \rightarrow b b X)$.

This is illustrated in Fig. 12, where we plot the ratio $N_{\text {edge }} / N_{\text {all }}$ as a function of $\operatorname{Br}($ edge $) / \operatorname{Br}(\tilde{g} \rightarrow b b X)$. Here we plot $N_{\text {edge }} / N_{\text {all }}$ instead of $N_{\text {fit }} / N_{\text {all }}$, because we have already seen $N_{\text {fit }} \sim N_{\text {edge }}$, and the statistical fluctuation of $N_{\text {edge }}$ is small ( $\sim 2 \%$ ) with a help of the generator information. Some points in the plots are away from the line $N_{\text {edge }} / N_{\text {all }}=\operatorname{Br}($ edge $) / \operatorname{Br}(\tilde{g} \rightarrow b b X)$ : The point $\mathrm{C}$ is off because the chargino has large branching ratios into leptons as discussed earlier. At the point T1, the 


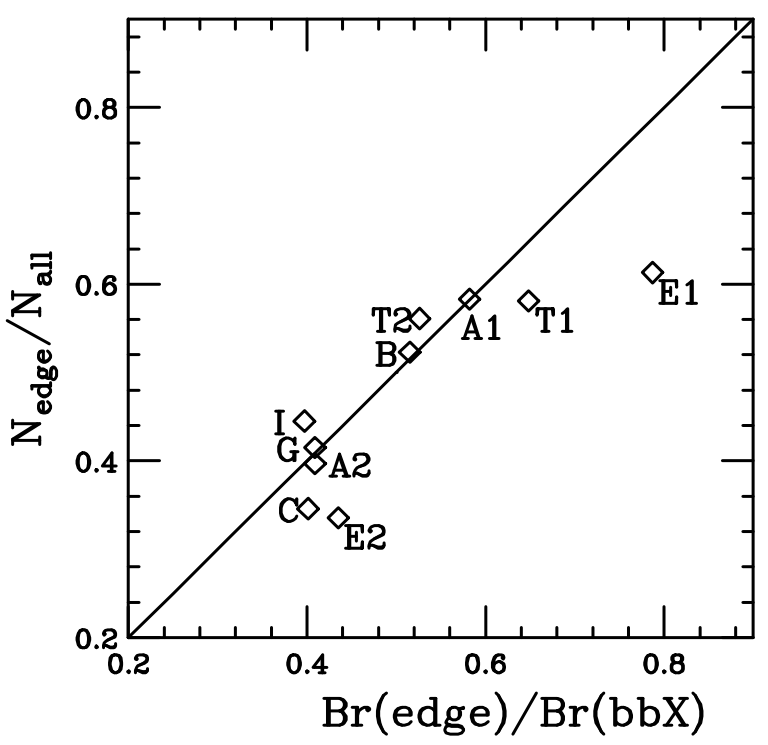

FIG. 12: Relation between $N_{\text {edge }} / N_{\text {all }}$ and $\operatorname{Br}($ edge $) / \operatorname{Br}(\tilde{g} \rightarrow$ $b b X)$.

$\tilde{t}_{1} \tilde{t}_{1}^{*}$ productions contributes to $N_{\text {all }}$. The points E1 and $\mathrm{E} 2$ are significantly off because the first and the second generation squarks dominantly decay into the gluino.

The systematics due to the chargino and neutralino decay patterns are difficult to estimate from the LHC data only. By combining the data from a future $e^{+} e^{-}$linear collider (LC) of $\sqrt{s}=500 \sim 1000 \mathrm{GeV}$ such systematics would be significantly reduced. Note that SUSY points with $m_{\tilde{\chi}_{1}^{+}} \lesssim 300 \mathrm{GeV}$ are studied in this paper, which is within the reach of a $\mathrm{TeV}$ scale LC.

If events with four $b$-jets dominate the SUSY events at the LHC, study of the events with four or three $b$ jets would be important, which is out of the scope of this paper. If $m_{\tilde{t}_{1}} \ll m_{\tilde{g}}$, it is important to constrain the stop mass in order to estimate the contribution from $\tilde{t}_{1} \tilde{t}_{1}^{*}$, as the cross section increases rapidly as $m_{\tilde{t}_{1}}$ decreases. The weighted end point $M_{t b}^{\mathrm{w}}$ would provide the information on $m_{\tilde{t}_{1}}$.

\section{E. Snowmass points}

In this subsection we check if we would reconstruct fake end points by any chance when the mode of our interest is insignificant. To this purpose, we generate a set of Monte Carlo data for the Snowmass points [18]. The Snowmass points and slopes (SPS) are a set of benchmark points and parameter lines in the MSSM parameter space corresponding to different scenarios in the search for SUSY at the present and future experiments. Some of those points correspond to the case where decay modes (III) or (IV) are not dominant, and we should not expect to see the edge structure in the $m_{t b}$ distribution.

We list the fit results at SPS 1-2, and 4-6 in Table VII. Here SPS 3 is not listed as it is the same as the point $\mathrm{C}$.
The $M_{t b}^{\text {fit }}$ is lower by $20 \mathrm{GeV}$ than the $M_{t b}^{\mathrm{w}}$ at SPS 1 and SPS 6, similar to the points discussed in Subsection III B (see Table V).

SPS 2 is so-called the "focus point", where the scalar mass at the GUT scale $m_{0}$ is larger than the GUT scale gaugino mass $M_{0}$. The gluino two body decays into a squark and a quark are not kinematically open, therefore the dominant decay modes of the gluino are the three body decays; $\operatorname{Br}\left(\tilde{g} \rightarrow t b \tilde{\chi}_{2}^{ \pm}\right)=25 \%, \operatorname{Br}\left(\tilde{g} \rightarrow t b \tilde{\chi}_{1}^{ \pm}\right)=$ $20 \%$, and $\operatorname{Br}\left(\tilde{g} \rightarrow t t \tilde{\chi}_{i}^{0^{\prime}} s\right)=20 \%$. The $m_{t b}$ distribution should not have any edge structure because it dominantly consists of the gluino three body decays. The reconstructed edge has only $4 \sigma$ in height, and is statistically insignificant. The $\chi^{2}$ value of the fit is also rather large, $\chi^{2}=44.7$ for 30 degrees of freedom [23]. The fitted end point $M_{t b}^{\mathrm{w}}=484.9 \pm 24.9 \mathrm{GeV}$ may be related to the mass difference between the gluino and the heavier chargino, $m_{\tilde{g}}=778.6 \mathrm{GeV}$ and $m_{\tilde{\chi}_{2}^{ \pm}}=296.3 \mathrm{GeV}$. The $m_{t b}$ distribution at SPS 2 is compared with that at SPS 1 in Fig. 13.

At SPS 5 , the stop is so light that the decay $\tilde{t}_{1} \rightarrow b \tilde{\chi}_{1}^{ \pm}$ is kinematically closed. The gluino decay branching ratio into $\tilde{b}_{1}$ is large $(9 \%)$, but the $\tilde{b}_{1}$ subsequently decays mostly into $W \tilde{t}_{1}(80 \%)$. We thus do not find any significant edge.

SPS 4 is an example that a straightforward application of our reconstruction technique fails to find $M_{t b}^{\mathrm{w}}$. The distribution is shown in Fig. 14. The sbottom is light because of large $\tan \beta$, and the dominant gluino decay mode is $\tilde{g} \rightarrow b \tilde{b}_{1}(78 \%)$. The sbottom further decays into chargino $(36 \%)$ or the second lightest neutralino $(30 \%)$. Decays of the chargino and the second lightest neutralino are dominated by $\tilde{\chi}_{1}^{ \pm} \rightarrow W \tilde{\chi}_{1}^{0}(100 \%)$ and $\tilde{\chi}_{2}^{0} \rightarrow Z^{0} \tilde{\chi}_{1}^{0}(98 \%)$, respectively. The dominance of the decay into $Z^{0}$ or $W$ is common when the decay into the Higgs boson or sleptons are kinematically forbidden. Some of the decays $\tilde{g} \rightarrow b \tilde{b} \rightarrow b b \tilde{\chi}_{2}^{0}$ are reconstructed as $t b$ events together with additional gauge bosons, resulting in the $m_{t b}$ distribution having a quasi-edge structure. The edge is reconstructed by our fit (see Table VII). Note that the end point of the $m_{b b}$ distribution of the decay is $403 \mathrm{GeV}$, while the reconstructed $m_{t b}$ distribution of the events with the decay $(\mathrm{I})_{2}$ has a peak around $500 \mathrm{GeV}$ and the distribution ends around $580 \mathrm{GeV}$, close to the kinematical limit $m_{\tilde{g}}-m_{\tilde{\chi}} \sim 600 \mathrm{GeV}$. The edge structure is identified as that of the mode $(\mathrm{I})_{2}$ with a tagged $Z^{0} \rightarrow l l$. In the $m_{t b}$ distribution, the second edge structure from the decay mode (III) or (IV) is weakly seen in the distribution. Assuming that two edges exist, we find the second end point $M_{t b}^{\text {fit }}=425.4 \pm 15.2 \mathrm{GeV}$ and $h=349.4 \pm 74.4 /(10 \mathrm{GeV})$.

\section{EVENTS WITH ADDITIONAL TAGS}

At the points given in Table I, the lighter chargino and neutralinos (inos) $\tilde{\chi}_{1}^{ \pm}, \tilde{\chi}_{1}^{0}$ and $\tilde{\chi}_{2}^{0}$ are gaugino-like, while the heavier inos $\tilde{\chi}^{h} \equiv \tilde{\chi}_{2}^{ \pm}, \tilde{\chi}_{3}^{0}$ and $\tilde{\chi}_{4}^{0}$ are higgsino- 

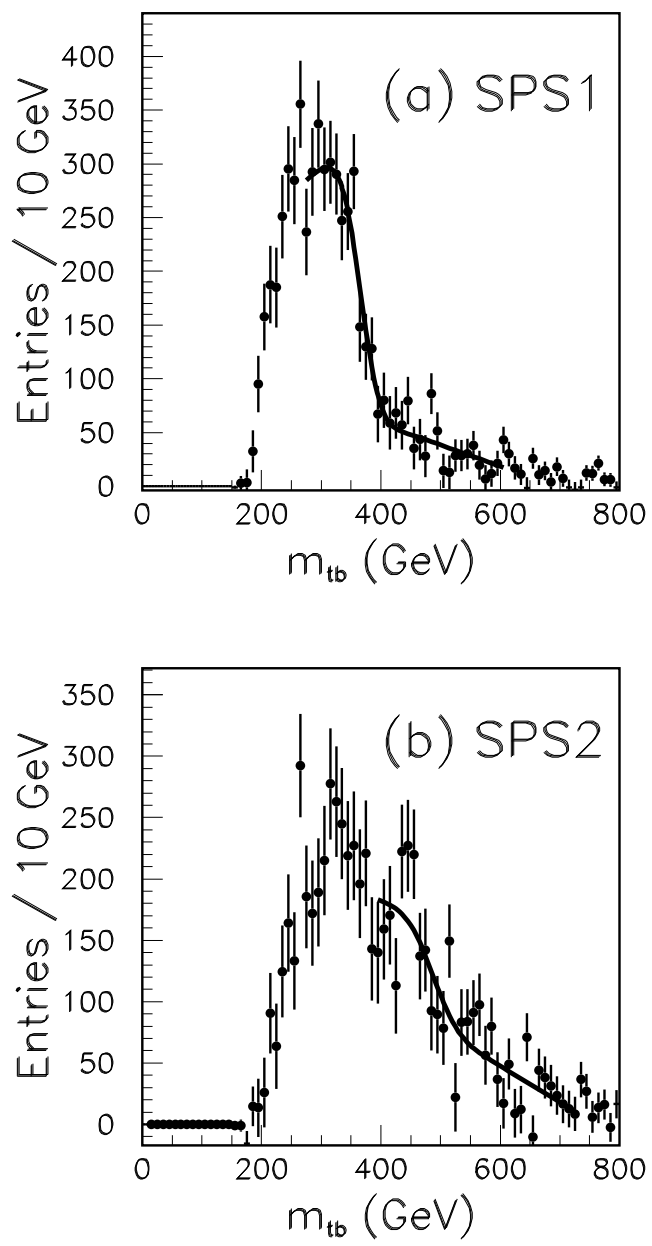

FIG. 13: The $m_{t b}$ distributions at (a) SPS 1 and (b) SPS 2. The fit curves are also shown.

\begin{tabular}{|l||rrr|}
\hline & $M_{t b}^{\mathrm{w}}[\mathrm{GeV}]$ & $M_{t b}^{\mathrm{fit}}[\mathrm{GeV}]$ & $h /(10 \mathrm{GeV})$ \\
\hline SPS 1 & 380.8 & $363.9 \pm 4.8$ & $267.3 \pm 20.8$ \\
SPS 2 & N/A & $484.9 \pm 24.9$ & $92.2 \pm 23.5$ \\
SPS 4 & 431.0 & $495.0 \pm 6.2$ & $515.7 \pm 33.0$ \\
SPS 5 & 416.2 & $442.6 \pm 42.8$ & $56.6 \pm 69.6$ \\
SPS 6 & 442.3 & $416.2 \pm 6.2$ & $326.4 \pm 24.7$ \\
\hline
\end{tabular}

TABLE VII: Fit results for the Snowmass points. Data sample at SPS 2 corresponds to $2 \times 10^{6}$ events, while other samples correspond to $3 \times 10^{6}$ events.

like. Unlike the first and second generation squarks, the third generation squarks can decay into the heavier inos. This is because the third generation squarks couple to the higgsinos by the top (or bottom) Yukawa coupling. The identification of the cascade decay chain $\tilde{g} \rightarrow \tilde{t} / \tilde{b} \rightarrow$ $\tilde{\chi}^{h}$ is a probe for the supersymmetric version of Yukawa interactions. In addition, the decay distribution may be

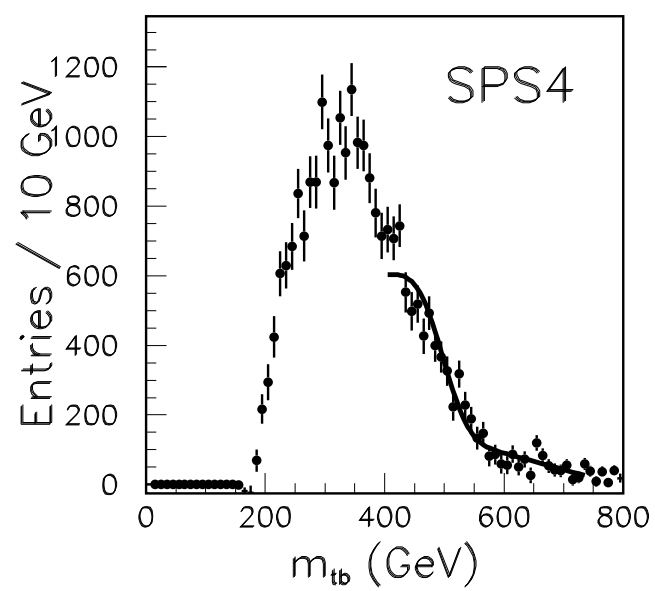

FIG. 14: The $m_{t b}$ distribution at SPS 4. The fit curve is also shown.

sensitive to the mass difference $m_{\tilde{g}}-m_{\tilde{\chi}^{h}} \sim M_{3}-\mu$.

In this section we show an example to select such events by requiring additional leptons in the final state. We study the point $\mathrm{C}$, where the stop and sbottom decay into $\tilde{\chi}^{h}$ with large branching fractions. At this point the gluino decay branching ratios are $\operatorname{Br}\left(\tilde{g} \rightarrow \tilde{t}_{1}\right)=15.1 \%$ and $\operatorname{Br}\left(\tilde{g} \rightarrow \tilde{b}_{1}\right)=14 \%$. The squarks further decay into the higgsino-like inos with branching ratios of $\operatorname{Br}\left(\tilde{b}_{1} \rightarrow\right.$ $\left.\tilde{\chi}^{h}\right)=24 \%$ and $\operatorname{Br}\left(\tilde{t}_{1} \rightarrow \tilde{\chi}^{h}\right)=13 \%$, respectively. The stop and sbottom decay branching ratios are comparable to those into $\tilde{\chi}_{1}^{ \pm}$.

Some of the decay branching ratios of the charginos and neutralinos are listed in Table VIII. The higgsinolike inos have large branching ratios into the $Z^{0}$ boson. The heavier inos also have non-negligible branching ratios into triple leptons, because they may also decay into $\tilde{\chi}_{2}^{0}$ which further decays into leptons.

The signature of the heavier inos from stop or sbottom is an excess of the $Z^{0}$ boson or three leptons in events with two $b$-jets. In Fig. 15 we plot the invariant mass of the same flavor opposite sign leptons $\left(m_{l l}\right)$ for the events with $n_{b}=0,1$ and 2 , where $n_{b}$ is the number of $b$-jets in an event. In the plots, accidental lepton pair distribution estimated with events with different-flavor opposite sign leptons are subtracted. All plots show a common structure that the invariant mass distribution increases toward the edges around $70 \mathrm{GeV}$ and $105 \mathrm{GeV}$, which correspond to the decay chains of $\tilde{\chi}_{2}^{0} \rightarrow l \tilde{l}_{L} \rightarrow l l \tilde{\chi}_{1}^{0}$ and $\tilde{\chi}_{2}^{0} \rightarrow l \tilde{l}_{R} \rightarrow l l \tilde{\chi}_{1}^{0}$, respectively. The $Z^{0}$ peak is also seen in the plots. As $n_{b}$ increases, the $Z^{0}$ peak height becomes more significant relative to the edge structure. The excess of $Z^{0} \rightarrow l l$ in the $n_{b}=2$ sample indicates the $Z^{0}$ boson originating primarily from stop or sbottom Qualitatively, the edges in the $m_{l l}$ distribution for the $n_{b}=2$ 


\begin{tabular}{|l|c|l|c|}
\hline decay mode & Br in $\%$ & decay mode & Br in $\%$ \\
\hline$\tilde{\chi}_{2}^{ \pm} \rightarrow W \tilde{\chi}_{2}^{0}$ & 29 & $\tilde{\chi}_{3}^{0} \rightarrow W \tilde{\chi}^{ \pm}$ & 59 \\
$\tilde{\chi}_{2}^{ \pm} \rightarrow Z^{0} \tilde{\chi}_{1}^{ \pm}$ & 26 & $\tilde{\chi}_{3}^{0} \rightarrow Z^{0} \tilde{\chi}_{1}^{0}$ & 1.7 \\
$\tilde{\chi}_{2}^{ \pm} \rightarrow l \tilde{\nu}$ & 3.0 & $\tilde{\chi}_{3}^{0} \rightarrow Z^{0} \tilde{\chi}_{2}^{0}$ & 25 \\
$\tilde{\chi}_{2}^{ \pm} \rightarrow \nu \tilde{l}$ & 6.8 & & \\
\hline$\tilde{\chi}_{4}^{0} \rightarrow W \tilde{\chi}_{1}^{ \pm}$ & 59 & $\tilde{\chi}_{2}^{0} \rightarrow l \tilde{l}_{L}$ & 9.8 \\
$\tilde{\chi}_{4}^{0} \rightarrow Z^{0} \tilde{\chi}_{1}^{0}$ & 1.7 & $\tilde{\chi}_{2}^{0} \rightarrow l \tilde{l}_{R}$ & 3.0 \\
$\tilde{\chi}_{4}^{0} \rightarrow Z^{0} \tilde{\chi}_{2}^{0}$ & 1.3 & $\tilde{\chi}_{1}^{ \pm} \rightarrow W \tilde{\chi}_{1}^{0}$ & 11 \\
$\tilde{\chi}_{4}^{0} \rightarrow l \tilde{l}_{L}$ & 3.0 & $\tilde{\chi}_{1}^{ \pm} \rightarrow l \tilde{\nu}_{L}$ & 37 \\
$\tilde{\chi}_{4}^{0} \rightarrow l \tilde{l}_{R}$ & 0.9 & $\tilde{\chi}_{1}^{ \pm} \rightarrow \nu \tilde{l}_{L}$ & 8.9 \\
\hline
\end{tabular}

TABLE VIII: Some of the chargino and neutralino branching ratios in $\%$ at the point $\mathrm{C}$.
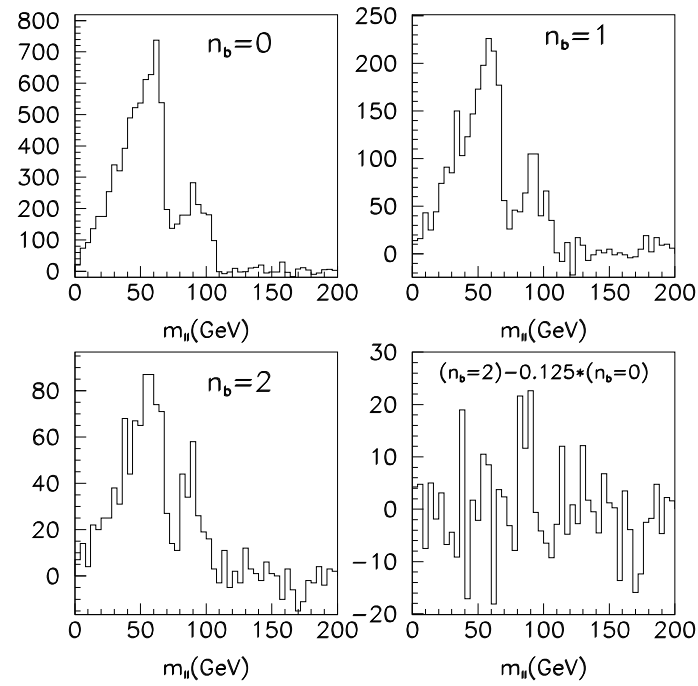

FIG. 15: Distributions of $m_{e^{+} e^{-}}+m_{\mu^{+} \mu^{-}}-m_{e^{+} \mu^{-}}-m_{e^{-} \mu^{+}}$ with $n_{b}=0$ (top-left), 1 (top-right), 2 (bottom-left). The bottom-right plot shows the distribution for $n_{b}=2$ after subtracting the scaled distribution for $n_{b}=0$ by a factor of 0.125 .

sample are completely subtracted by the distribution for the $n_{b}=0$ sample scaled by a factor of 0.125 , and the $Z^{0}$ peak remains (the bottom-right plot of Fig. 15).

The same analysis can be performed by using events with three leptons. In Fig. 16 we plot sum of invariant mass distributions; $m_{e^{+} e^{-}}+m_{\mu^{+} \mu^{-}}-m_{e^{+} \mu^{-}}-m_{e^{-} \mu^{+}}$for all possible combinations of opposite sign lepton pairs. In this mode the $Z^{0}$ peak is more significant. The ratio of the edge heights in the $m_{l l}$ distribution for the $n_{b}=$ 2 sample to that of the $n_{b}=0$ sample is 0.25 . This means that the events originating from the decay $\tilde{b} / \tilde{t} \rightarrow$ $\tilde{\chi}^{h}$ dominate the three lepton events, and the events with $n_{b}=0$ arise due to misidentified $b$-jets.

Note that these plots at the point $\mathrm{C}$ are made for a total of $3 \times 10^{6}$ SUSY events, which corresponds to an integrated luminosity of $600 \mathrm{fb}^{-1}$. The statistics for 100 $\mathrm{fb}^{-1}$ is not significant. However, this procedure should be useful for lighter mass spectrum where the decay of
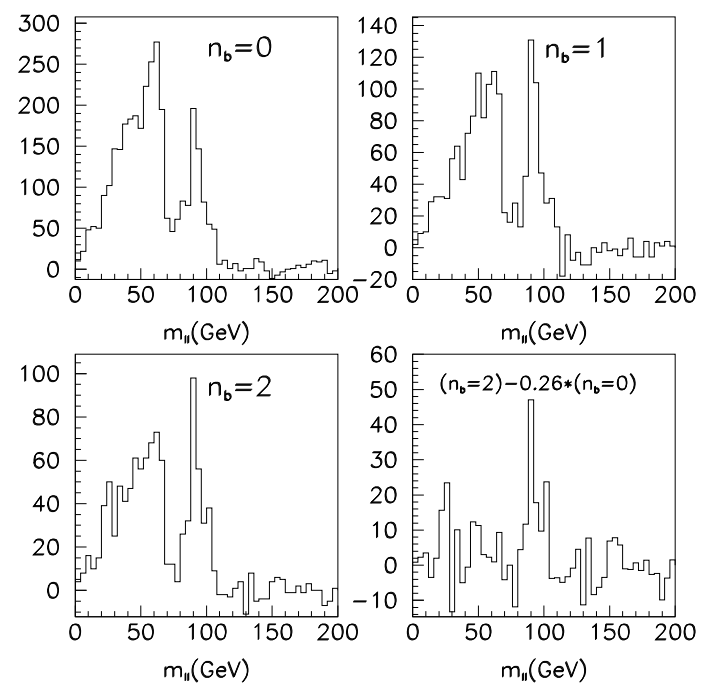

FIG. 16: Same as Fig. 15, but for events with three leptons.

the third generation squarks to $\tilde{\chi}^{h}$ is open.

After establishing the contribution of the decay $\tilde{t} / \tilde{b} \rightarrow$ $\tilde{\chi}^{h}$ in the tagged $b b 3 l$ and $b b Z^{0}$ events, we may reconstruct the $t b$ system in the sample. The $t b$ reconstruction is similar to that described in Section II, except that the distributions are made without the cuts for the effective mass and the lepton, because the standard model background is expected to be small now. In Fig. 17 the $m_{t b}$ distribution with tagged three leptons is shown. The number of events after the sideband subtraction is 242.5 . The $m_{t b}$ distribution is concentrated just below the expected end point which is close to $m_{\tilde{g}}-m_{\tilde{\chi}_{2}^{ \pm}} \sim 400 \mathrm{GeV}$, although the statistics is low. The distribution of tagged $Z^{0} \rightarrow l l$ is similar and the number of events after the sideband subtraction is 142 .

\section{STOP AND SBOTTOM PROPERTIES IN THE MSUGRA MODEL}

In this section we interpret our study of the third generation squarks at the LHC in a framework of the MSUGRA model. The LHC may determine the MSUGRA parameter $m_{0}, M_{0}, A_{0}$, and $\tan \beta$ through the measurement of various distributions. The decay chains $\tilde{q} \rightarrow q \tilde{\chi}_{2}^{0}$ followed by $\tilde{\chi}_{2}^{0} \rightarrow h \tilde{\chi}_{1}^{0}, Z^{0} \tilde{\chi}_{1}^{0}, l \tilde{l}$, or $l l \tilde{\chi}_{1}^{0}$ are especially useful if the kinematical end points of the distributions are measured. The effective mass measurement of the inclusive SUSY signal is also useful to determine the absolute SUSY scale. Among these parameters, the $A_{0}$ parameter is only weakly constrained. This is because the left-right mixings of sbottom and stau are almost fixed by $\mu \tan \beta$, and $A_{t}$ is insensitive to $A_{0}$ as will be described below. One of the possible ways to determine $A_{0}$ is to measure the mass difference between squarks 

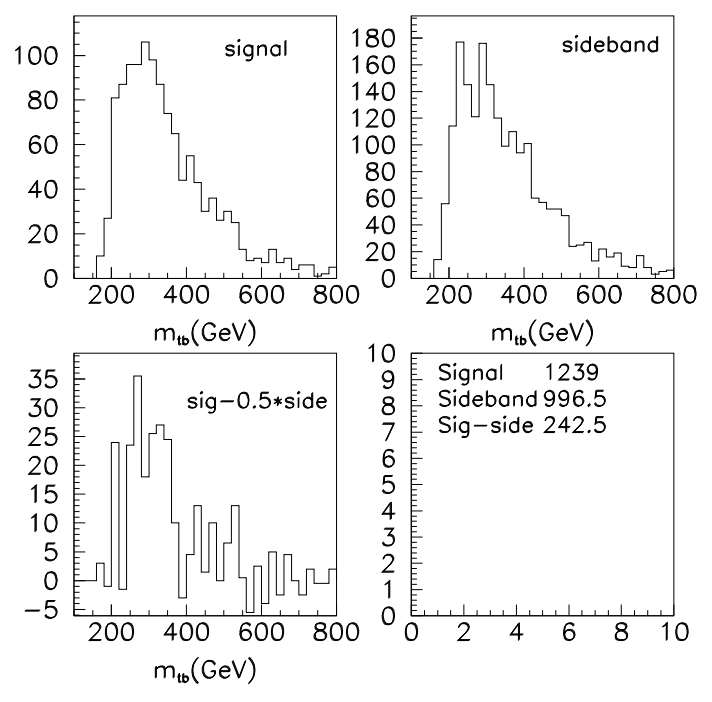

FIG. 17: The $m_{t b}$ distributions for events with tagged three leptons. Top-left: signal distribution. Top-right: background estimated by the sideband events. Bottom-left: distribution after the sideband subtractions.

or sleptons with different flavors, which is caused by the renormalization group equation (RGE) running between the GUT scale and the weak scale.

The squark-mass matrix is given as follows,

$$
-\mathcal{L}_{\text {mass }}=\left(\begin{array}{cc}
\tilde{f}_{L}^{\star} & \tilde{f}_{R}^{\star}
\end{array}\right)\left(\begin{array}{cc}
m_{L L}^{2} & m_{L R}^{2} \\
m_{L L}^{2} & m_{R R}^{2}
\end{array}\right)\left(\begin{array}{c}
\tilde{f}_{L} \\
\tilde{f}_{R}
\end{array}\right),
$$

where

$$
\begin{aligned}
& m_{L L}^{2}=m_{\tilde{t}_{L}}^{2}+m_{t}^{2}+m_{Z}^{2} \cos 2 \beta\left(\frac{1}{2}-\frac{2}{3} \sin ^{2} \theta_{W}\right), \\
& m_{R R}^{2}=m_{\tilde{t}_{R}}^{2}+m_{t}^{2}+\frac{2}{3} m_{Z}^{2} \cos 2 \beta \sin ^{2} \theta_{W} \\
& m_{L R}^{2}=m_{t}\left(A_{t}-\mu \cot \beta\right)
\end{aligned}
$$

for stops, and

$$
\begin{aligned}
& m_{L L}^{2}=m_{\tilde{t}_{L}}^{2}+m_{b}^{2}+m_{Z}^{2} \cos 2 \beta\left(-\frac{1}{2}+\frac{1}{3} \sin ^{2} \theta_{W}\right) \\
& m_{R R}^{2}=m_{\tilde{b}_{R}}^{2}+m_{b}^{2}-\frac{1}{3} m_{Z}^{2} \cos 2 \beta \sin ^{2} \theta_{W} \\
& m_{L R}^{2}=m_{b}\left(A_{b}-\mu \tan \beta\right)
\end{aligned}
$$

for sbottoms.

In the MSUGRA, the SUSY-breaking parameters at the weak scale in these mass matrices are evaluated by the RGEs with the universal GUT scale boundary conditions. When the bottom-Yukawa coupling is negligible, it is convenient to present the SUSY-breaking parameters by the infrared-fixed point value for the top-Yukawa coupling constant $\bar{y}_{f}[19]$,

$$
\bar{y}_{f}=\frac{F^{\prime}(t)}{6 F(t)} .
$$

Here,

$$
F(t)=\int_{0}^{t} d t^{\prime}\left(\frac{\alpha_{3}(t)}{\alpha_{G U T}}\right)^{\frac{16}{9}}\left(\frac{\alpha_{2}(t)}{\alpha_{G U T}}\right)^{-3}\left(\frac{\alpha_{1}(t)}{\alpha_{G U T}}\right)^{-\frac{13}{99}}
$$

where $t=1 /(4 \pi) \log \mu^{2} / M_{G U T}^{2}$ and $\mu$ is the renormalization scale. $\alpha_{G U T}$ is the gauge coupling constant at the GUT scale. The SUSY breaking parameters for the third generation at the low energy scale are approximately given using the value as

$$
\begin{aligned}
m_{\tilde{t}_{L}}^{2} \simeq & 0.7 M_{\tilde{g}}^{2}+0.5 m_{0}^{2}-\left(0.16 A_{0}^{2}-0.25 A_{0} M_{\tilde{g}}\right)(1-\xi) \\
m_{\tilde{t}_{R}}^{2} \simeq & 0.5 M_{\tilde{g}}^{2}+m_{0}^{2}(1-\xi) \\
& -\left(0.33 A_{0}^{2}-0.51 A_{0} M_{\tilde{g}}\right)(1-\xi) \\
m_{\tilde{b}_{R}}^{2} \simeq & m_{0}^{2}+0.8 M_{\tilde{g}}^{2} \\
A_{t} \simeq & -0.7 M_{\tilde{g}}+A_{0}(1-\xi), \\
A_{b} \simeq & -1.2 M_{\tilde{g}}+0.8 A_{0}
\end{aligned}
$$

up to $\mathcal{O}\left((1-\xi)^{2}\right)$, where

$$
\xi\left(\equiv y_{t}^{2} / \bar{y}_{t}^{2}\right)=\left(\frac{m_{t}}{203 \sin \beta(\mathrm{GeV})}\right)^{2} .
$$

Here we use the GUT relation for the gaugino masses, $M_{\tilde{g}} \simeq 2.8 M_{0}$. For the pole top mass $m_{t}^{\text {pole }}=175 \mathrm{GeV}, \xi$ ranges $0.7 \lesssim \xi \lesssim 0.9$.

From Eq. (15), the qualitative behavior of the SUSYbreaking parameters is clear. The reduction of the $m_{\tilde{t}_{L}}^{2}$ and $m_{\tilde{t}_{R}}^{2}$ is more efficient for $A_{0} M_{\tilde{g}}<0$, although the dependence is suppressed by an overall factor of $1-\xi$. The right handed stop mass $m_{\tilde{t}_{R}}$ is also insensitive to $m_{0}$. In a moderate $\tan \beta$ region, $\tilde{t}_{1}$ and $\tilde{b}_{1}$ is almost right-handed and left-handed, respectively.

In Fig. 18 we show the masses of $\tilde{g}, \tilde{t}_{1}$, and $\tilde{b}_{1}$ as functions of $A_{0}$, where the other MSUGRA parameters are fixed to be $m_{0}=230 \mathrm{GeV}, M_{0}=300 \mathrm{GeV}, \tan \beta=10$, and $\mu>0$. As expected from the discussion above, the masses $m_{\tilde{t}_{1}}$ and $m_{\tilde{b}_{1}}$ have sensitivity to $A_{0}$. The reduction of $m_{\tilde{t}_{L}}^{2}$ and $m_{\tilde{t}_{R}}^{2}$ by the top-Yukawa coupling constant is larger when $A_{0}<0$.

The stop $\tilde{t}_{1}$, which is almost $\tilde{t}_{R}$, is lighter than the gluino unless $m_{0}$ is very large. Similarly, since the sbottom $\tilde{b}_{1}$ is almost $\tilde{b}_{L}$, the branching ratio to the edge events in Eq. (1), which includes $\tilde{\chi}_{1}^{ \pm}$, is large since it has $\mathrm{SU}(2)$ gauge and top-Yukawa interactions. The study of the edge events has therefore potentially high feasibility in the MSUGRA. Fig. 19 shows the regions, where the gluino decays into $t \tilde{t}_{1}$ and $b \tilde{b}_{1}$ are open, in a plane of $m_{0}$ and $A_{0}$. In this plot the other parameters are fixed to be $m_{\tilde{g}}=707 \mathrm{GeV}, \tan \beta=5$ or 30 , and $\mu>0$.

Since the top quark mass deviates from the fixed-point value, the $A_{0}$ dependence on $m_{\tilde{t}_{L}}^{2}$ and $m_{\tilde{t}_{R}}^{2}$ survives, as shown in Fig. 18. This may lead to a measurable dependence on $A_{0}$ of the end point of the $m_{t b}$ distribution. In Fig. 20(a), we show $M_{t b}^{\mathrm{w}}, M_{t b}(\mathrm{III})_{1}$, and $M_{t b}(\mathrm{IV})_{11}$ as functions of $A_{0}$. Since $\tilde{b}_{1}$ is heavy, $M_{t b}(\mathrm{IV})_{11}$ is lower 


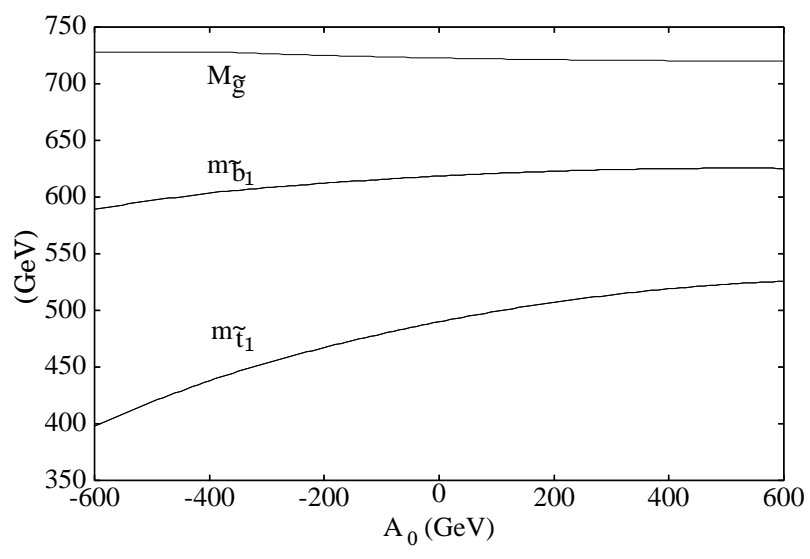

FIG. 18: Masses for $\tilde{g}, \tilde{t}_{1}$, and $\tilde{b}_{1}$ as functions of $A_{0}$. Here, $m_{0}=230 \mathrm{GeV}, M_{0}=300 \mathrm{GeV}, \tan \beta=30$, and $\mu>0$.

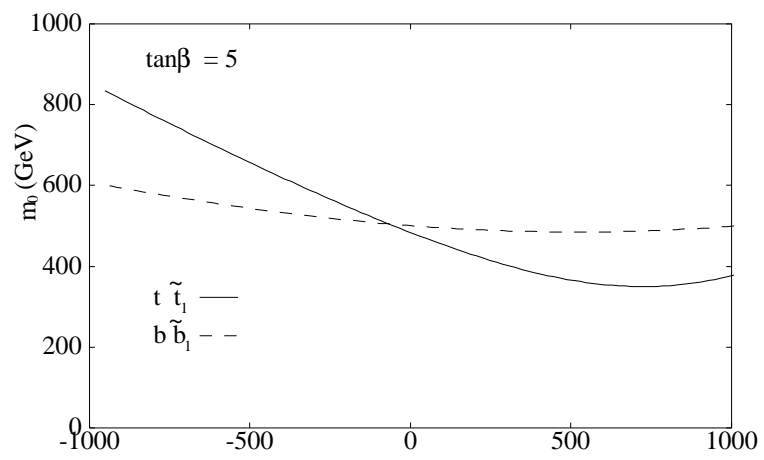

$\mathrm{A}_{0}(\mathrm{GeV})$

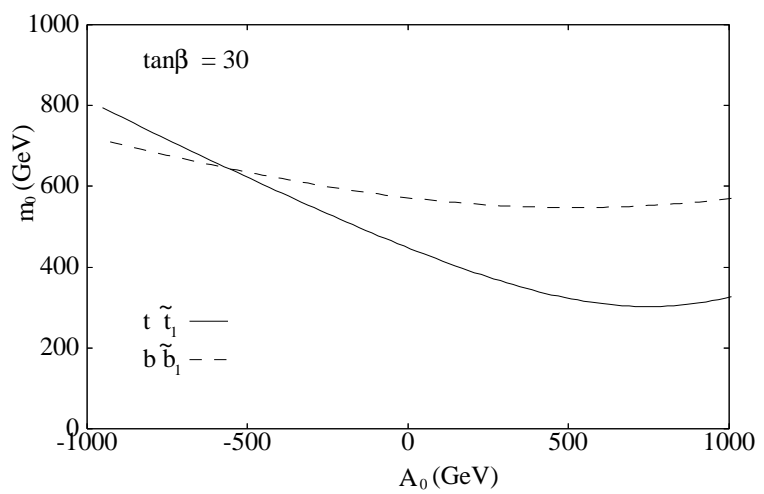

FIG. 19: Contours where $m_{\tilde{g}}=m_{\tilde{t}_{1}}+m_{t}$ and $m_{\tilde{b}_{1}}+m_{b}$ on a plane of $m_{0}$ and $A_{0}$. The gluino decays into $t \tilde{t}_{1}$ and $b \tilde{b}_{1}$ are open below the contours. Here, $m_{\tilde{g}}=707 \mathrm{GeV}, \tan \beta=5$ and 30 , and $\mu>0$.

than $M_{t b}(\mathrm{III})_{1}$. However, $\operatorname{Br}\left(\tilde{g} \rightarrow t \tilde{t}_{1}\right)$ is larger than $\operatorname{Br}\left(\tilde{g} \rightarrow b \tilde{b}_{1}\right)$ due to the phase space, which makes $M_{t b}^{\mathrm{w}}$ closer to $M_{t b}(\mathrm{III})_{1}$. If $M_{t b}^{\mathrm{w}}$ is determined with a precision of a few $\%$ as in our simulation study, the $A_{0}$ parameter can be evaluated with a precision of $\sim 50 \mathrm{GeV}$.

In addition to the weighted end point $M_{t b}^{\mathrm{w}}$, the edge height $h$ of the $m_{t b}$ distribution is measurable, and the height is roughly proportional to the branching ratio of the gluino to the edge events arising from the decay modes (III) and (IV) in Eq. (1). In Subsection III D we proposed to investigate $N_{\text {fit }} / N_{\text {all }}$ where $N_{\text {fit }}$ is the number of the edge event estimated by the fit and $N_{\text {all }}$ is the total $t b$ events selected. It is related to the normalized branching ratio $\operatorname{Br}($ edge $) / \operatorname{Br}(\tilde{g} \rightarrow b b X)$. The normalized branching ratio is sensitive to the branching ratios for $\tilde{t}_{1}$ and $\tilde{b}_{1}$, and uncertainty on fragmentation and $b$-tagging efficiency would be canceled out in the ratio.

In Fig. 20(b) we show the branching ratio $\operatorname{Br}(\tilde{g} \rightarrow b b X)$ and the branching ratio of the gluino to the edge events normalized by $\operatorname{Br}(\tilde{g} \rightarrow b b X)$. They are decreasing functions of $A_{0}$, since the $\tilde{t}_{1}$ and $\tilde{b}_{1}$ is heavier for larger $A_{0}$. While the behavior of $\operatorname{Br}(\tilde{g} \rightarrow b b X)$ is moderate, the normalized branching ratio of the edge events is sensitive to $A_{0}$, since it depends on the branching ratios of $\tilde{t}_{1}$ and $\tilde{b}_{1}$. In Fig. 20(b) the normalized branching ratio has two kinks, where some decay modes for $\tilde{t}_{1}$ or $\tilde{b}_{1}$ are open. The first kink around $A_{0} \sim-500 \mathrm{GeV}$ comes from a change of the branching ratio of $\tilde{t}_{1}$. The dominant decay modes of $\tilde{t}_{1}$ are $b \tilde{\chi}_{1}^{ \pm}$and $t \tilde{\chi}_{1}^{0}$, however, the mode $t \tilde{\chi}_{2}^{0}$ is open around $A_{0} \sim-500 \mathrm{GeV}$. The decay modes $\tilde{t}_{1} \rightarrow b \tilde{\chi}_{2}^{ \pm}$and $\tilde{b}_{1} \rightarrow t \tilde{\chi}_{2}^{ \pm}$are open at the second kink around $A_{0} \sim-150 \mathrm{GeV}$. The decay modes are not included in the edge events, because the end point $M_{t b}(\mathrm{III})_{2}$ is significantly lower than $M_{t b}(\mathrm{III})_{1}$. The additional tags for $\tilde{\chi}_{2}^{ \pm}$discussed in the previous section to identify the decay works for the region. Since $\tilde{\chi}_{2}^{ \pm}$is higgsino-like and the Yukawa coupling to $\tilde{b}_{1}$ is enhanced by the top-Yukawa coupling, the decay mode $\tilde{b}_{1} \rightarrow t \tilde{\chi}_{2}^{ \pm}$ becomes dominant for $A_{0}>-150 \mathrm{GeV}$, and then the normalized branching ratio become quickly diluted.

In the MSUGRA, the masses of $\tilde{t}_{1}$ and $\tilde{b}_{1}$ are related with each other in a broad parameter space. As the determination of $m_{\tilde{b}_{1}}$ has been studied in Ref. [2], we now discuss $M_{t b}^{\mathrm{w}}$ and the normalized branching ratio with a fixed $m_{\tilde{b}_{1}}$ value. We fix $m_{\tilde{b}_{1}}=570 \mathrm{GeV}$ and $M_{\tilde{g}}=707 \mathrm{GeV}$ for $\tan \beta=5$ and 30 , and show $M_{t b}^{\mathrm{w}}\left(M_{t b}(\mathrm{III})_{1}\right)$ as a function of $m_{\tilde{t}_{1}}$ in Fig. 21(a). Our parameter scan is restricted to the range $\left|A_{0}\right|<2 \mathrm{TeV}$.

We have two disconnected solutions corresponding to $-1400(-1850) \mathrm{GeV}<A_{0}<-280$ (470) $\mathrm{GeV}$ and 1350 (1600) $\mathrm{GeV}<A_{0}<2000 \mathrm{GeV}$ for $\tan \beta=5$ (30), respectively. The $A_{0}$ region between the two solutions is not allowed because of the experimental constraint of $\tilde{\tau}$ mass or charged LSP. For $\tan \beta=5(30), m_{0}$ is smaller than $460(750) \mathrm{GeV}$ for $A_{0}<0$, and 500 (300) GeV for $A_{0}>0$. Since $\tilde{b}_{1} \sim \tilde{b}_{L}$ in the MSUGRA, a larger $\left|A_{0}\right|$ corresponds to a larger $m_{0}$ for a fixed $m_{\tilde{b}_{1}}$, as expected from Eq. (15). Nevertheless $\tilde{t}_{1}$ could be much lighter than $\tilde{b}_{1}$ for a large and negative value of $A_{0}$ as can be seen in Fig. 21(a) [24]. Note that $M_{t b}^{\mathrm{w}}$ is determined for a fixed $\tilde{t}_{1}$ mass when $m_{\tilde{t}_{1}}$ is lighter than $370 \mathrm{GeV}$. If $m_{\tilde{b}_{1}}$ is constrained elsewhere, $m_{\tilde{t}_{1}}$ is strongly restricted by the $M_{t b}^{\mathrm{w}}$ measurement under the MSUGRA assumption.

In Fig. 21(b) we show the solution in a $M_{t b}^{\mathrm{w}}$ and $\operatorname{Br}($ edge $) / \operatorname{Br}(\tilde{g} \rightarrow b b X)$ plane. The normalized branching ratios is almost 1 for $M_{t b}^{\mathrm{w}}<400 \mathrm{GeV}$ (or $m_{\tilde{t}_{1}}<$ 

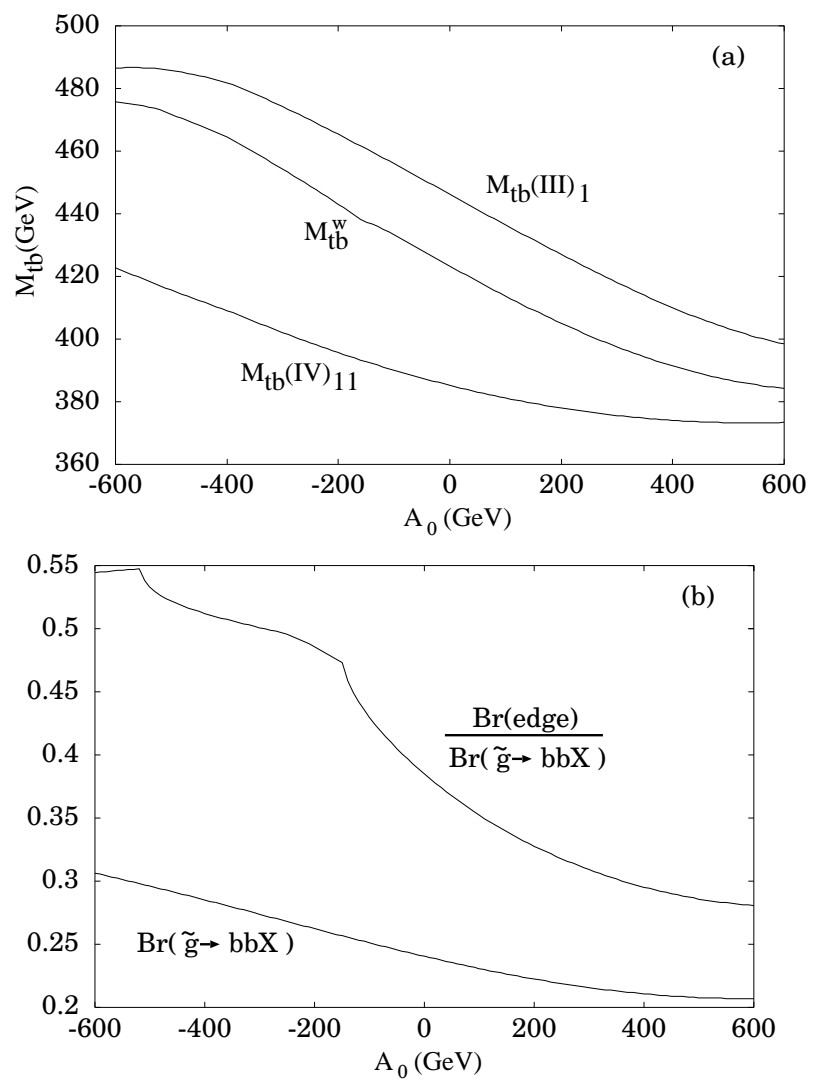

FIG. 20: (a) $M_{t b}^{\mathrm{w}}, M(\mathrm{III})_{1}$, and $M(\mathrm{IV})_{11}$ as functions of $A_{0}$. (b) $\operatorname{Br}(\tilde{g} \rightarrow b b X)$ and $\operatorname{Br}($ edge $) / \operatorname{Br}(\tilde{g} \rightarrow b b X)$ as functions of $A_{0}$. Input parameters are the same as in Fig. 18.

$300 \mathrm{GeV})$. When the mass difference of $m_{\tilde{t}_{1}}$ and $m_{\tilde{b}_{1}}$ is large, the decay of $\tilde{b}_{1}$ is dominated by $W \tilde{t}_{1}$. Furthermore, for $m_{\tilde{t}_{1}} \lesssim 300 \mathrm{GeV}, \operatorname{Br}\left(\tilde{t}_{1} \rightarrow b \tilde{\chi}_{1}^{ \pm}\right)$is $100 \%$. However since $m_{\tilde{q}}>m_{\tilde{g}}$ in this region, squark and gluino production goes to the final states having four bottom quarks, where we have seen the disagreement between the measurement $N_{\text {edge }} / N_{\text {all }}$ and $\operatorname{Br}($ edge $) / \operatorname{Br}(\tilde{g} \rightarrow b b X)$ in Section III D. In this case our study must be extended to events with more than three tagged $b$-jets.

Note that the decay $\tilde{\chi}_{2}^{0} \rightarrow \tilde{l}$ is mostly closed in Fig. 21 . The decay is open only at the most right region of Fig. 21(b), near the end of the lines of the solutions. If this decay is open, the masses of $\tilde{q}, \tilde{l}$ and $\tilde{\chi}_{1}^{0}$ are modelindependently determined by the $m_{l l}, m_{l l j}$ and $m_{l j}$ distributions. When this decay mode is kinematically forbidden, one needs to combine various distributions for consistency check of the MSUGRA assumptions. As is already shown in Fig. 19, the decay $\tilde{g} \rightarrow \tilde{b} / \tilde{t}$ is open up to $m_{0} \sim m_{\tilde{g}}$, providing information of the third generation squarks in the wide region of the parameter space.
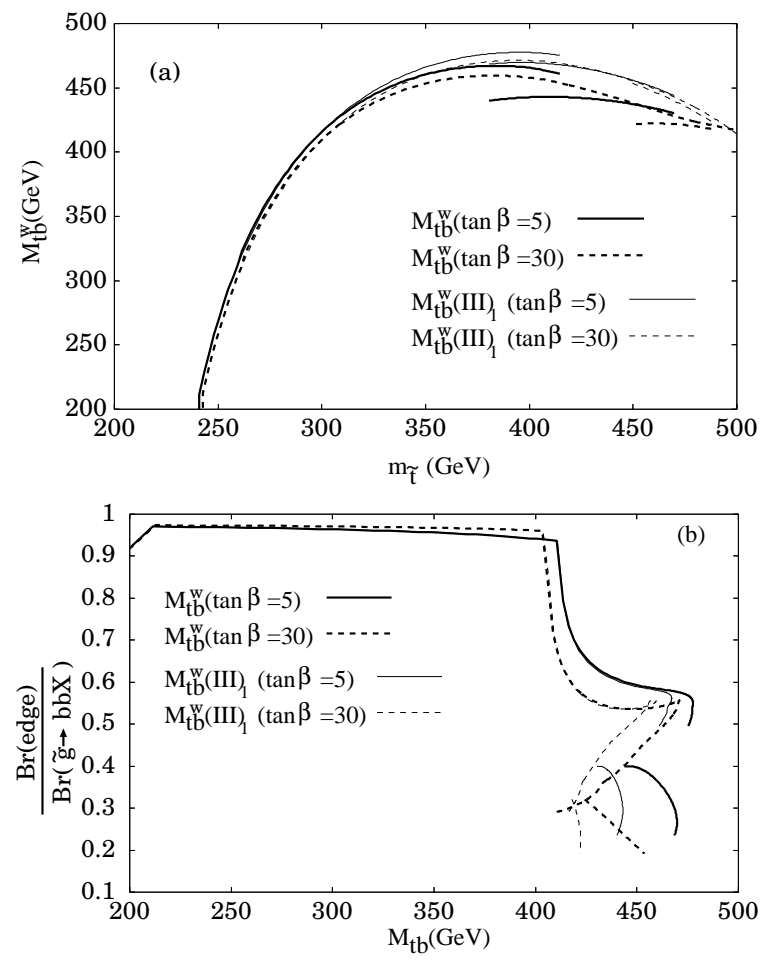

FIG. 21: For fixed $m_{\tilde{b}_{1}}=570 \mathrm{GeV}$ and $M_{\tilde{g}}=707 \mathrm{GeV}$, (a) $M_{t b}^{\mathrm{w}}$ and $m_{\tilde{t}_{1}}$, and (b) $M_{t b}^{\mathrm{w}}\left(M_{t b}(\mathrm{III})\right)$ and $\operatorname{Br}($ edge $) / \operatorname{Br}(\tilde{g} \rightarrow$ $b b X)$. Here, $-2 \mathrm{TeV}<A_{0}<2 \mathrm{TeV}, \tan \beta=5$ and 30 , and $\mu>0 . m_{0}$ is fixed by $m_{\tilde{b}_{1}}$. The neutralino LSP is assumed.

\section{MEASUREMENT OF TOP POLARIZATION}

Similar to the tau-lepton decay, we may measure the polarization of the top quark since it decays to $b W$ via the $(V-A)$ interaction. The top quarks from the $\tilde{g}, \tilde{t}$, and $\tilde{b}$ decays are polarized, and the polarization depends on the mixing angles for stops, charginos, and neutralinos.

The bottom quark angular distribution in the polarized top quark decay is the following,

$$
\frac{1}{\Gamma_{t}} \frac{d \Gamma_{t}}{d \cos \theta} \propto\left(\frac{m_{t}}{m_{W}}\right)^{2} \sin ^{2} \frac{\theta}{2}+2 \cos ^{2} \frac{\theta}{2},
$$

where $\theta$ is the angle between the direction of the bottom quark and the direction of the top quark spin in the rest frame of the top quark. The terms proportional to $\left(m_{t} / m_{W}\right)^{2}$ come from the decay to the longitudinal $W$ boson. The bottom quark thus tends to go to the opposite direction of the top quark spin.

In the decay mode (III) ${ }_{1}$, the top quark from a gluino decay is polarized to be left-handed (right-handed) if $\tilde{t}_{1}$ is left-handed (right-handed). The polarization is reflected on the invariant mass distribution of the $b b$ system $\left(m_{b b}\right)$, if the top quark is relativistic enough in the gluino rest frame. Here, one of the bottom quarks comes from the top quark decay and the other comes from $\tilde{t}_{1} \rightarrow b \tilde{\chi}^{ \pm}$. Especially, when the invariant mass $m_{t b}$ is close to the end point $M_{t b}$ of the decay mode (III) $)_{1}$, the top and bottom 


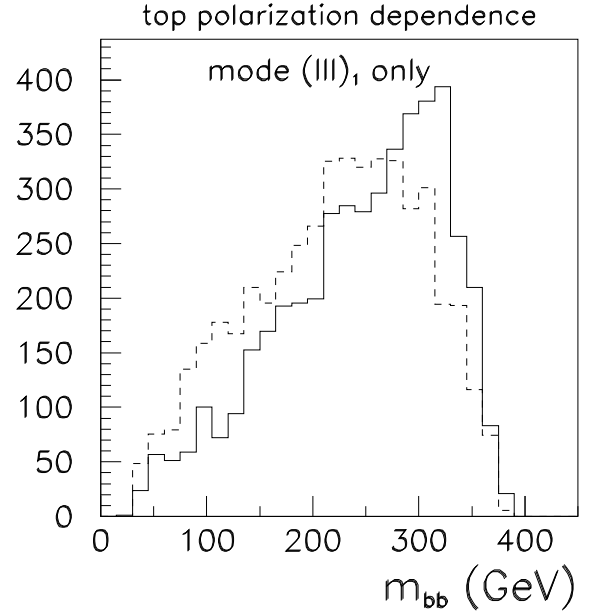

FIG. 22: Distribution of $m_{b b}$ in the decay chain (III) $)_{1}$. The (dashed) line is for $\tilde{t}_{1}=\tilde{t}_{L}\left(\tilde{t}_{R}\right)$, and $400 \mathrm{GeV}<m_{t b}<$ $470 \mathrm{GeV}$. We use the mass spectrum in the sample point A1 in Table I, and the normalization is arbitrary.

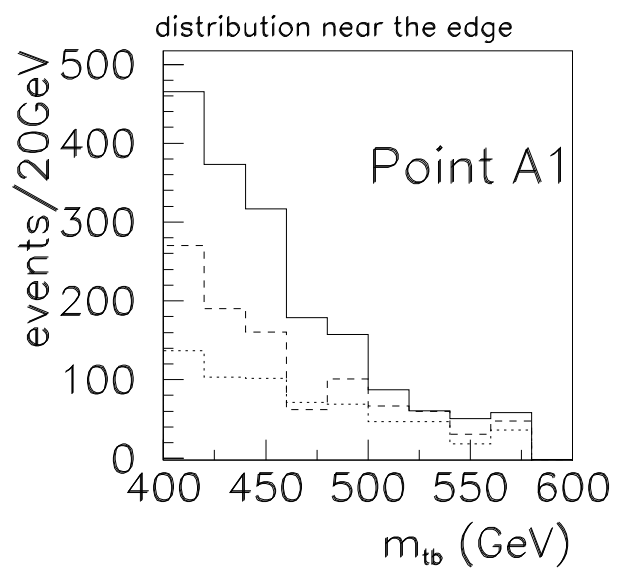

FIG. 23: The solid line is for the total $m_{t b}$ distribution at the sample point $\mathrm{A} 1$, the dashed line is for the $m_{t b}$ distribution excluding the mode (III) $)_{1}$, and the dotted line is for the $m_{t b}$ distribution excluding the modes (III) $1,(\text { III })_{11}$ and (III) $)_{21}$.

quarks go to the opposite direction to each other in the gluino rest frame. Thus, the distribution of the invariant mass $m_{b b}$ for events with $m_{t b}$ close to $M_{t b}(\mathrm{III})_{1}$ is harder (softer) for left-handed (right-handed) top quarks.

In Fig. 22 we show the $m_{b b}$ distribution from the decay chain (III) $)_{1}$. In this simulation, we use the HERWIG generator, since it respects helicities for each particles in the processes. We generated a large number of events which go through the decay (III) $)_{1}$. We use the mass spectrum at the reference point $\mathrm{A} 1$ in Table I. We use events with $400 \mathrm{GeV}<m_{t b}<470 \mathrm{GeV}$ to make the distribution, and the solid (dotted) line is for the left-handed (righthanded) stop. The statistical significance in the difference between the left-handed and right-handed stops is about $3 \sigma$ for $\mathcal{O}(100)$ events.

In the above simulation, we neglect contribution of other decay chains such as (III) $)_{11}$ and (IV $)_{11}$. The other decay chains may contribute to the $m_{b b}$ distribution even if we impose that $m_{t b}$ is near the end point. The $m_{b b}$ distributions in the modes (III) $)_{11}$ and (IV) ${ }_{11}$ do not depend on the polarization for the top quark, since the top quark distribution in the scalar boson decay is spherical. In Fig. 23 we show the $m_{t b}$ distribution around the edge region at the reference point $\mathrm{A} 1$. The solid line is for the total distribution, the dashed line is for the distribution excluding the mode (III) $)_{1}$, and the dotted line is for the distribution excluding the modes (III) $)_{1},(\mathrm{III})_{11}$, and $(\mathrm{III})_{21}$. About a half of the events near the end point come from the signal mode (III) $)_{1}$, and the ratio between the signal events and the rest depends on the MSSM parameters.

\section{CONCLUSIONS}

In this paper we study cascade decays $\tilde{g} \rightarrow$ $\left(t \tilde{t}_{1}\right.$ or $\left.b \tilde{b}_{i}\right) \rightarrow t b \tilde{\chi}_{i}^{ \pm}$at the LHC by reconstructing $t b$ final state where the top quark decays hadronically. The $m_{t b}$ distribution of the cascade decay has an edge structure. The measurement of the end point and the edge height of the $m_{t b}$ distribution constrains a combination of the masses of $\tilde{g}, \tilde{b}, \tilde{t}$ and $\tilde{\chi}^{ \pm}$and the decay branching ratios of the particles involved in the decays.

Through a detailed simulation study, we show in this paper the measurement of the end point and edge height on a continuum background is indeed possible. Namely, the end point of the cascade decay calculated in parton level agrees with the reconstructed edge position, and the ratio of $N_{\text {edge }}$ (the number of reconstructed edge events) and $N_{\text {all }}$ (the number of total reconstructed $t b$ events) is understood well by the ratio $\operatorname{Br}($ edge $) / \operatorname{Br}(\tilde{g} \rightarrow b b X)$.

The end point and branching ratios depend on the mass and left-right mixing of the $\tilde{t}$ and $\tilde{b}$, as well as the chargino and neutralino masses and mixings in the MSSM. In the MSUGRA, these sparticle spectrum is expressed by a few parameters at the GUT scale. The decay mode $\tilde{g} \rightarrow\left(t \tilde{t}_{1}\right.$ or $\left.b \tilde{b}_{i}\right) \rightarrow t b \tilde{\chi}_{1}^{ \pm}$is open for a wide region of the parameter space where $m_{0}<m_{\tilde{g}}$. The $m_{t b}$ distribution is sensitive to the $A_{0}$ parameter, the trilinear coupling at the GUT scale. The distribution is most sensitive to $A_{0}$ when $A_{0} \cdot M_{0}<0$.

The stop and sbottom could decay both into the heavier and lighter charginos and neutralinos unlike the first and second generation squarks. This is because the third generation squarks have the large top (bottom)-Yukawa coupling to the higgsinos. A strategy to search for such 
decays specific for the stop and sbottom is described in Section IV. The Yukawa coupling is also related to the $\tilde{t}_{L}-\tilde{t}_{R}$ mixing. The polarization of the top quark arising from the gluino decay $\tilde{g} \rightarrow t \tilde{t}$ depends on the stop leftright mixing. The dependence of the $m_{b b}$ distribution on the top polarization in the tagged $t b$ sample is discussed in Section VI.

To understand the event distribution better, one needs to know the nature of the quark and gluon fragmentations into jets. The reconstruction efficiencies are significantly different between the two standard SUSY generators HERWIG and PYTHIA, which adopt different models for the fragmentation. We point out that the smearing of the jet-pair invariant mass originating from a $W$ decay affects the reconstruction efficiency.

The interplay between the LHC and the future LC would be useful to reduce the systematics coming from the uncertainties of sparticle masses and decay branching ratios. They would be reduced dramatically if some of the charginos and neutralinos are accessible at the LC. Model independent and precise determination of the stop and sbottom masses may be possible in such cases and will be discussed elsewhere.

\section{Acknowledgment}

We thank the ATLAS collaboration members for useful discussion. We have made use of the physics analysis framework and tools which are the result of collaboration-wide efforts. We especially thank Dr. Kanzaki and Mr. Toya. We acknowledge ICEPP, Univ. of Tokyo, for providing us computing resources. This work is supported in part by the Grant-in-Aid for Science Research, Ministry of Education, Science and Culture, Japan (No.13135297 and No.14046225 for JH, No.11207101 for KK, and No.14540260 and 14046210 for MMN).

\section{Appendix: Reliability of Sideband Subtraction}

In this paper, we generate events both by the PYTHIA and HERWIG generators. The HERWIG generator uses a parton-shower approach for initial- and final- state QCD radiations including the color coherence effects and the azimuthal correlation both within and between jets [13]. The full available phase space for the parton shower is restricted to an angular order region, namely, the angle between the two emitted partons is smaller than that of previous branches. On the other hand, the PYTHIA generator adopts the string model [12]. The two generators predict different $t b$ reconstruction efficiencies, which may be considered as the uncertainty in fragmentation.

Definition of jets also affects the number of reconstructed $t b$ events and the reconstruction efficiency. We try two algorithms, a cone-based algorithm and a $K_{T}$ algorithm (Montreal version), available in the JET Finder

\begin{tabular}{|rr|cc|}
\hline gen & jet & $M_{t b}^{\text {fit }}[\mathrm{GeV}]$ & $h /(10 \mathrm{GeV})$ \\
\hline PY & cone 0.4 & $455.2 \pm 8.2$ & $271.4 \pm 22.7$ \\
& 0.5 & $436.1 \pm 7.7$ & $272.9 \pm 33.4$ \\
& 0.6 & $461.1 \pm 10.4$ & $217.8 \pm 22.8$ \\
& $K_{T} 0.4$ & $442.0 \pm 4.7$ & $321.3 \pm 22.3$ \\
& 0.5 & $452.2 \pm 6.0$ & $305.3 \pm 21.3$ \\
0.6 & $459.1 \pm 6.1$ & $241.6 \pm 31.6$ \\
\hline HW cone 0.4 & $434.5 \pm 5.8$ & $354.8 \pm 23.3$ \\
& 0.5 & $460.2 \pm 4.9$ & $349.2 \pm 22.8$ \\
0.6 & $440.9 \pm 7.1$ & $305.3 \pm 33.7$ \\
& $K_{T} 0.4$ & $434.9 \pm 4.3$ & $406.5 \pm 22.1$ \\
& 0.5 & $460.0 \pm 5.5$ & $379.6 \pm 33.5$ \\
0.6 & $468.4 \pm 5.8$ & $314.3 \pm 20.7$ \\
\hline
\end{tabular}

TABLE IX: Fit results at the point A1. We use the conebased algorithm or the $K_{T}$ algorithm with cone sizes of $R=$ $0.4,0.5,0.6$. The fit is based on $3 \times 10^{6}$ events. The $M_{t b}^{\mathrm{w}}$ is $459 \mathrm{GeV}$.

Library [20], which is interfaced to the ATLFAST packages.

The cone-based algorithm merges the calorimeter cells around a high $E_{T}$ cell in a fixed cone size $R$. On the other hand, in the $K_{T}$ algorithm, a cell called a protojet $i$ grows until there are no more protojets $j$ which satisfy $(\Delta \eta)^{2}+(\Delta \phi)^{2}<\left(E_{T i}^{2} / \min \left(E_{T i}^{2}, E_{T j}^{2}\right)\right) R^{2}$, therefore the cone size depends on $E_{T}$ 's, and the shape of the jet depends on the distribution of the particles in the jet. The $K_{T}$ algorithm is based on QCD, and it is considered to be advantageous to merge soft jets from an initial parton, although experimentally challenging.

The fit results with different generators and jet finding algorithms at the point A1 is summarized in Table IX. The end point $M_{t b}^{\mathrm{fit}}$ becomes closer to $M_{t b}^{\mathrm{w}}$ as $R$ increases. This is expected because more soft jets are merged to leading jets as $R$ increases. The edge height $h$ is larger for HERWIG with the $K_{T}$ algorithm. The difference of the height for $R \leq 0.5$ is more than $25 \%$ in Table IX. The height significantly decreases for $R=0.6$. This is because we have to reconstruct a jet pair with relatively small invariant mass $\sim 80 \mathrm{GeV}$.

To see the origin of the difference more closely, we compare the $m_{j j}$ distributions consistent to the top interpretation in Fig. 24, where, the solid histograms are the invariant mass distributions for jet pairs which satisfy $\left|m_{j j}-m_{W}\right|<15 \mathrm{GeV}$ and $\left|m_{b j j}-m_{t}\right|<30 \mathrm{GeV}$. Dashed histograms show the distribution of accidental jet pairs in the $W$ mass region, that is, the "fake $W$ " background, estimated by the sideband method. The left plots are the distributions of the events arising from the decay chain (III) ${ }_{1}$, while the right plots are the $m_{t b}$ distribution of all selected $t b$ events. The distribution at the bottom-left (HERWIG and the $K_{T}$ algorithm for $R=0.5$ ) are more concentrated around $m_{j j} \sim 80 \mathrm{GeV}$ compared to the others (PYTHIA and the cone-based or the $K_{T}$ algorithm), corresponding to better reconstruction efficiencies. 


\begin{tabular}{|c|c|c|c|c|c|}
\hline$K_{T} /$ cone & $\mathrm{R}$ & $\begin{array}{r}\text { HW } \\
\text { sig }\end{array}$ & sub & $\begin{array}{c}\text { PY } \\
\text { sig } \\
\end{array}$ & er sub \\
\hline \multicolumn{6}{|l|}{$\operatorname{mode}(\text { III })_{1}$} \\
\hline cone & 0.4 & 10180 & 2949 & 9695 & 2462 \\
\hline cone & 0.5 & 9659 & 2974 & 9213 & 2447 \\
\hline$K_{T}$ & 0.4 & 9405 & 3105 & 9226 & 2813 \\
\hline$K_{T}$ & 0.5 & 9118 & 3239 & 8607 & 2808 \\
\hline \multicolumn{6}{|l|}{$\operatorname{mode}(\mathrm{I})_{2}$} \\
\hline cone & 0.4 & 2779 & 574 & 2630 & 500 \\
\hline$K_{T}$ & 0.5 & 2086 & 448 & 2058 & 433 \\
\hline
\end{tabular}

TABLE X: Numbers of events before and after the sideband subtraction for the events with a gluino that decays through mode $(\mathrm{III})_{1} \tilde{g} \rightarrow t \tilde{t}_{1} \rightarrow t b \tilde{\chi}_{1}^{ \pm}$and mode (I) $)_{2} \tilde{g} \rightarrow b \tilde{b}_{1} \rightarrow b b \tilde{\chi}_{2}^{0}$.

Incompleteness of the sideband subtraction is seen in the same figure. The sideband subtraction uses the jet pairs with invariant mass $35 \mathrm{GeV}<m_{j j}<65 \mathrm{GeV}$ and $95 \mathrm{GeV}<m_{j j}<125 \mathrm{GeV}$ to estimate the "fake W" background. On the other hand, as the jets are defined to have $p_{T}^{\text {jet }}>10 \mathrm{GeV}$, a jet pair near $m_{j j} \sim 35 \mathrm{GeV}$ is suppressed. We see an underestimate of background events in the range $65 \mathrm{GeV}<m_{j j}<70 \mathrm{GeV}$, where the dotted line and solid line differ significantly. We find that events with $m_{j j}<70 \mathrm{GeV}$ do not contribute to the edge structure of the $m_{t b}$ distribution. The events in this region might have to be removed from the candidate events.

The incompleteness of the sideband subtraction can also be seen by comparing the reconstruction efficiency of the events from specific decay chains. Here we take two decay chains for comparison;

$$
\begin{aligned}
(\mathrm{III})_{1} & \tilde{g} \rightarrow t \tilde{t}_{1} \rightarrow t b \tilde{\chi}_{1}^{ \pm}, \\
(\mathrm{I})_{2} & \tilde{g} \rightarrow b \tilde{b}_{1} \rightarrow b b \tilde{\chi}_{2}^{0} .
\end{aligned}
$$

The decay chain (III) $)_{1}$ is relevant to the $t b$ events. On the other hand, as the decay chain $(\mathrm{I})_{2}$ has no $W$, the sideband subtraction should remove the events arising from the decay chain completely, unless there are accidental weak bosons, or the understimation of the misreconstructed events that has just been discussed above.

The branching ratio $\operatorname{Br}\left(\tilde{\chi}_{2}^{0} \rightarrow Z^{0} \tilde{\chi}_{1}^{0}\right)$ is small $(1.5 \%)$ at the point A1. The decay branching ratios at the point A1 are

$$
\begin{aligned}
\operatorname{Br}(\mathrm{III})_{1} & =\operatorname{Br}\left(\tilde{g} \rightarrow t \tilde{t}_{1}\right) \operatorname{Br}\left(\tilde{t}_{1} \rightarrow b \tilde{\chi}_{1}^{ \pm}\right) \times 0.7 \\
& =0.078 \\
\operatorname{Br}(\mathrm{I})_{2} & =\operatorname{Br}\left(\tilde{g} \rightarrow b \tilde{b}_{1}\right) \operatorname{Br}\left(\tilde{b}_{1} \rightarrow b \tilde{\chi}_{2}^{0}\right) \\
& =0.025
\end{aligned}
$$

where the factor 0.7 is the hadronic branching ratio of the $W$ boson.

The numbers of reconstructed $t b$ events before and after the sideband subtraction are listed in Table X. For the $K_{T}$ algorithm with $R=0.5$, the ratio of the numbers $\mathrm{N}(\mathrm{I})_{2} / \mathrm{N}(\mathrm{III})_{1}$ are 0.229 and 0.15 before and after the sideband subtraction, respectively. Comparing these ratios to that of the branching ratios $\operatorname{Br}(\mathrm{I})_{2} / \operatorname{Br}(\mathrm{III})_{1}=0.32$,
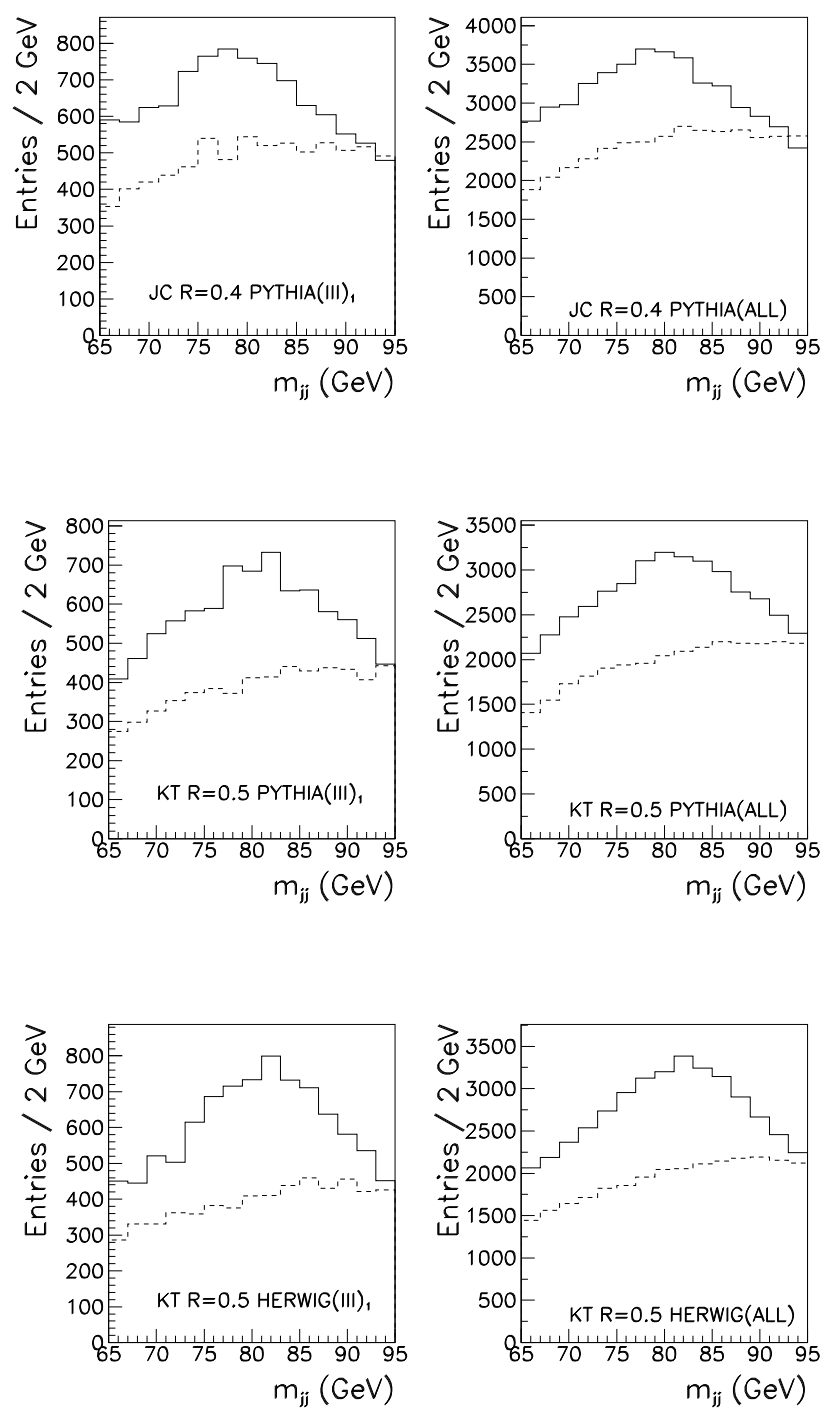

FIG. 24: Distributions of $m_{j j}$ consistent to the $W$ boson mass under the cuts described in Section II. The MC data is generated for the point A1. In the left, we plot the events that contain the decay chain $\tilde{g} \rightarrow t \tilde{t}_{1} \rightarrow t b \tilde{\chi}_{1}^{ \pm}$only, while we plots all events in the right. Dotted lines show estimated background. Top figures: PYTHIA with the cone-based algorithm $(R=0.4)$. Middle: PYTHIA with the $K_{T}$ algorithm $(R=0.5)$. Bottom: HERWIG with the $K_{T}$ algorithm $(R=0.5)$.

we see that the requirement $\left|m_{b j j}-m_{t}\right|<30 \mathrm{GeV}$ reduces the contribution of $(\mathrm{I})_{2}$ to about $70 \%$ relative to (III) ${ }_{1}$ and it is further reduced to less than $50 \%$ after the sideband subtraction. This ratio may be improved further by cutting the events with $m_{j j}<70 \mathrm{GeV}$ or by using more sophisticated background estimation. 
[1] See references in H. P. Nilles, Phys. Rept. 110, 1 (1984).

[2] ATLAS: Detector and physics performance technical design report, CERN-LHCC-99-14.

[3] R. Barbieri, G. R. Dvali and L. J. Hall, Phys. Lett. B 377, 76 (1996).

[4] M. Dine, A. Kagan and S. Samuel, Phys. Lett. B 243, 250 (1990); S. Dimopoulos and G. F. Giudice, Phys. Lett. B 357, 573 (1995); A. Pomarol and D. Tommasini, Nucl. Phys. B 466, 3 (1996); A. G. Cohen, D. B. Kaplan and A. E. Nelson, Phys. Lett. B 388, 588 (1996); J. Hisano, K. Kurosawa and Y. Nomura, Phys. Lett. B 445, 316 (1999): Nucl. Phys. B 584, 3 (2000).

[5] A.E. Nelson and M.J. Strassler, JHEP 0009, 030 (2000); T. Kobayashi and H. Terao, Phys. Rev. D 64, 075003 (2001); T. Kobayashi, H. Nakano, and H. Terao, Phys. Rev. D 65, 015006 (2002); T. Kobayashi, H. Nakano, T. Noguchi and H. Terao, Phys. Rev. D 66, 095011 (2002); JHEP 0302, 022 (2003).

[6] M. Carena and C. E. Wagner, Nucl. Phys. B 452, 45 (1995).

[7] H. E. Haber and R. Hempfling, Phys. Rev. Lett. 66, 1815 (1991); Y. Okada, M. Yamaguchi and T. Yanagida, Prog. Theor. Phys. 85, 1 (1991); J. R. Ellis, G. Ridolfi and F. Zwirner, Phys. Lett. B 257, 83 (1991); R. Barbieri and M. Frigeni, Phys. Lett. B 258, 395 (1991).

[8] R. Barbieri and G. F. Giudice, Phys. Lett. B 309, 86 (1993).

[9] I. Hinchliffe, F. E. Paige, M. D. Shapiro, J. Soderqvist and W. Yao, Phys. Rev. D 55, 5520 (1997).

[10] J. Hisano, K. Kawagoe, R. Kitano and M. M. Nojiri, Phys. Rev. D 66, 115004 (2002).

[11] H. Baer, F. E. Paige, S. D. Protopopescu and X. Tata "ISAJET 7.48: a Monte Carlo event generator for $p p, \bar{p} p$, and $e^{+} e^{-}$reactions", hep-ph/0001086.

[12] T. Sjostrand, L. Lonnblad and S. Mrenna "PYTHIA 6.2: physics and manual", hep-ph/0108264.

[13] G. Corcella, I.G. Knowles, G. Marchesini, S. Moretti, K. Odagiri, P. Richardson, M.H. Seymour and B.R. Webber, JHEP 0101 (2001) 010.

[14] E.Richter-Was et al., ATLFAST2.21, ATLAS Internal Note PHYS-NO-079.

[15] M. Battaglia et al., Eur. Phys. J. C 22, 535 (2001).

[16] J. Hisano, R. Kitano and M. M. Nojiri, Phys. Rev. D 65, 116002 (2002).

[17] D.R. Tovey Phys. Lett. B 4981 (2001), ATLAS NOTE ATL-PHYS-2002-013, Eur. Phys. J. direct C4: N4 2002.

[18] B. C. Allanach et al., in Proc. of the APS/DPF/DPB Summer Study on the Future of Particle Physics (Snowmass 2001) ed. N. Graf, Eur. Phys. J. C 25, 113 (2002).

[19] W. A. Bardeen, M. Carena, S. Pokorski and C. E. Wagner, Phys. Lett. B 320, 110 (1994).

[20] "Jet Finder Library: version 1.0" M. Bosman et. al. ATLSOFT-98-038.

[21] We do not consider a gluino decay into $t \tilde{t}_{2}$ for this paper.

[22] Larger $R$ values, $R=0.5$ and 0.6 are also tried to check the dependence on $R$ of the cone-based algorithm. For comparison, another jet reconstruction algorithm, the $K_{T}$ algorithm, was also tried with $R=0.4,0.5,0.6$, and 0.7. The comparison is given in the Appendix.

[23] However, $\Delta \chi^{2}$ is sometimes large even when the decay mode (III) dominates the distribution. For example $\Delta \chi^{2}=41.4$ for the point A1.

[24] $\left|A_{0}\right|<3 m_{0}$ for $A_{0}<0$, satisfying a condition for the consistent minimum for the potential. 